\title{
Enhancement of superconductivity by an external magnetic field in magnetic alloys
}

\author{
Dawid Borycki ${ }^{\mathrm{a}}$ \\ Institute of Physics, Faculty of Physics, Astronomy and Informatics, Nicolaus Copernicus University, Grudziadzka 5, \\ 87-100 Torun, Poland
}

Received 23 August 2013 / Received in final form 5 April 2014

Published online 21 May 2014

(c) The Author(s) 2014. This article is published with open access at Springerlink.com

\begin{abstract}
An infinite-volume limit solution of the thermodynamics of a BCS superconductor containing spin $1 / 2$ and $7 / 2$ magnetic impurities, obtained recently in [D. Borycki, J. Maćkowiak, Supercond. Sci. Technol. 24, 035007 (2011)] is exploited to derive the expressions for critical magnetic field $\mathcal{H}_{\mathrm{c}}(T)$. The credibility of the resulting thermodynamically limited theoretical equations, which depend on the magnetic coupling constant $g$ and impurity concentration $c$, is verified on the experimental data for the following superconducting alloys: LaCe, ThGd and $\mathrm{SmRh}_{4} \mathrm{~B}_{4}$. Good quantitative agreement with experimental data is found for sufficiently small values of $c$. The discrepancies between theoretical and experimental values of $\mathcal{H}_{\mathrm{c}}(T)$ for larger values of $c$ in case of LaCe and ThGd are reduced by introducing the concept of the effective temperature $\tilde{T}$, which accounts for the Coulomb interactions between the electron gas and impurity ions. At low temperatures, the critical magnetic field is found to increase with decreasing temperature $T$. This enhancement of the critical magnetic field provides evidence of the Jaccarino-Peter effect, which was experimentally observed in the Kondo systems like LaCe, $\left(\mathrm{La}_{1-x} \mathrm{Ce}_{x}\right) \mathrm{Al}_{2}$ and also in the pseudoternary compounds, including $\mathrm{Sn}_{1-x} \mathrm{Eu}_{x} \mathrm{Mo}_{6} \mathrm{~S}_{8}, \mathrm{~Pb}_{1-x} \mathrm{Eu}_{x} \mathrm{Mo}_{6} \mathrm{~S}_{8}$ and $\mathrm{La}_{1.2-x} \mathrm{Eu}_{x} \mathrm{Mo}_{6} \mathrm{~S}_{8}$. The effect of an external magnetic field $\mathcal{H}$ on a BCS superconductor perturbed by magnetic impurities was also studied. On these grounds, by analyzing the dependence of superconducting transition temperature $T_{\mathrm{c}}$ on $\mathcal{H}$ of $\left(\mathrm{La}_{1-x} \mathrm{Ce}_{x}\right) \mathrm{Al}_{2}$, we have shown, that for certain parameter values, external magnetic field compensates the destructive effect of magnetic impurities.
\end{abstract}

\section{Introduction}

The effect of superconductivity has been discovered over 100 years ago [1]. Since then, many extraordinary properties accompanying this phenomenon, including perfect diamagnetism, zero-resistance, magnetic levitation due to expulsion of the magnetic field from a superconductor, flux quantization, Josephson effect and vortex state have been explained.

The promising future applications of superconductors mainly concern electric power applications [2] and generation of high, uniform magnetic field, e.g. for magnetic resonance imaging purposes [3]. However, the wide-scale applications of superconductors are limited, because superconductivity has been proven to be very sensitive to the destructive effect of an external magnetic field and the current density. In order to extend the applications of superconductors it is strongly desirable to enhance the values of the critical magnetic field, critical currents and superconducting transition temperature. These topics were of high interest during past few decades [4-15].

The superconducting critical temperature, as shown by Bednorz and Müller [4] and later by Hosono group [5],

\footnotetext{
a e-mail: dawid.borycki@fizyka.umk.pl
}

can be raised by adding new elements to the antiferromagnetically ordered host system. Superconductivity appears at small value of dopant concentration $x$. Subsequently, the transition temperature $T_{\mathrm{c}}$ increases almost linearly with $x$, and after reaching a maximum at optimal doping level $x_{o p t}$, decreases and finally falls to zero. According to this scenario, the existence of parent magnetic correlations is viewed to be an essential feature of high-temperature superconductivity.

On the other hand, Matthias et al. [6] discovered that the superconducting transition temperature of lanthanum decreases, when small amount (1 at.\%) of the rare-earth magnetic impurities are added. It was shown, that the depression of $T_{\mathrm{c}}$ increases with the impurity spin value and not, as it was expected, with the impurity magnetic moment.

The experimental studies carried out by Matthias et al. revealed another extraordinary property of superconducting alloys, namely the possible coexistence of magnetism and superconductivity. The coexistence of superconductivity and long-range antiferromagnetic ordering of the rare earth $\mathrm{R}$ magnetic moments was later discovered in $\mathrm{RMo}_{6} \mathrm{Se}_{8}\left(\mathrm{R}=\mathrm{Gd}\right.$, Tb and Er) [16], $\mathrm{RRh}_{4} \mathrm{~B}_{4}(\mathrm{R}=\mathrm{Nd}$, $\mathrm{Sm}$ and $\mathrm{Tm})$ [17] and in $\mathrm{RMo}_{6} \mathrm{~S}_{8}(\mathrm{R}=\mathrm{Gd}, \mathrm{Tb}, \mathrm{Dy}$ 
and Er) [18]. A similar overlap between superconductivity and ferromagnetism was observed in $\mathrm{ErRh}_{4} \mathrm{~B}_{4}$ [19] and $\mathrm{HoMo}_{6} \mathrm{~S}_{8}[20]$.

This discovery was surprising, since magnetism and superconductivity had been believed to be mutually exclusive, because the internal magnetic fields generated in magnetically ordered systems are much larger than the typical critical fields of superconductors. However, as independently predicted by Fulde and Ferrell [21] and Larkin and Ovchinnikov [22], the superconductor may overcome the pair-breaking effect of magnetic field by forming periodic regions of superconductivity separated by domains of aligned spins. In such FFLO-state the order parameter is spatially modulated along the field direction. There are strong experimental suggestions for the occurrence of FFLO state in some heavy-fermion compounds, e.g. in $\mathrm{CeCoIn}_{5}$ [23].

Paradoxically, under specific circumstances, the external magnetic field can even enhance (instead of depress) the properties of superconductors, e.g. the upper critical field $\mathcal{H}_{\mathrm{c} 2}$ of $\mathrm{SmRh}_{4} \mathrm{~B}_{4}$ increases below the Néel temperature $T_{\mathrm{N}}=0.87 \mathrm{~K}[9,10]$. The latter can be understood on the grounds of the Jaccarino-Peter effect [24], in which the external magnetic field compensates the antiferromagnetic exchange interaction generated by the conduction electrons antiferromagnetically coupled to the spins of localized magnetic moments. The Jaccarino-peter compensation effect has been observed in several systems, e.g. in $\mathrm{Eu}_{0.75} \mathrm{Sn}_{0.25} \mathrm{Mo}_{6} \mathrm{~S}_{7.2} \mathrm{Se}_{0.8}[25]$.

In this study, we shall not investigate the FFLO state, since we assume a spatially uniform superconducting order parameter and spherical Fermi surfaces. In such the case, only the Jaccarino-Peter effect can be considered as the possible source of the superconductivity enhancement. Before going into details, let us briefly summarize theoretical investigations of the superconductors containing magnetic impurities.

Early theoretical investigations of the problem of magnetic superconducting alloys were founded on perturbation theory. Nakamura [26] and Suhl and Matthias [27] explained this effect by treating the $s$ - $d$ interaction $V_{s-d}[28]$ as an additive term in the total Hamiltonian, which perturbs a BCS superconductor [29]. Balseiro and Falicov [30] studied a BCS superconductor perturbed by magnetic impurities interacting via a nearest neighbor Heisenberg potential. The resulting phase diagrams comply qualitatively with experiment.

The well-known Abrikosov-Gor'kov theory [31] (AG) of dirty superconductors explains the strong decrease in $T_{\mathrm{c}}$ due to magnetic impurities and also predicts gapless superconductivity, confirmed experimentally by Reif and Woolf [32]. Disagreement with this approach is observed in a number of Kondo superconductors, e.g. $\mathrm{La}_{1-x} \mathrm{Ce}_{x} \mathrm{Al}_{2}$ [12], LaCe and LaGd [33] and PbCe and $\mathrm{InCe}[34]$.

The AG theory was generalized to describe an increase of $\mathcal{H}_{\mathrm{c} 2}$ below the Néel temperature $T_{\mathrm{N}}$. To this end, Ramakrishnan and Varma [35] extended AG theory to the case, when the magnetic ions are present in large con- centration. They solved numerically Eliashberg equations including the effects of phonons, spin waves and elastic scattering in order to estimate the variations of the pairbreaking parameter. However, a detailed comparison of their results with experimental data for the upper critical field of $\mathrm{SmRh}_{4} \mathrm{~B}_{4}$ have been not performed.

Hamaker et al. [36] found a good fit of the expression for the upper critical magnetic field in Machida's theory for antiferromagnetic superconductors [37] to their experimental data on $\mathrm{SmRh}_{4} \mathrm{~B}_{4}$ and obtained good quantitative agreement. However, some of the adjustable parameter values does not comply with other experimental findings.

The effect of magnetic impurities on superconductivity is still under debate. Soto and others [38] have experimentally discovered that the superconducting fluctuation effects above $T_{\mathrm{c}}$ in lanthanum are enhanced by praseodymium impurities. Kozorezov et al. [39] have shown, that trace concentrations of magnetic impurities may also result in significant changes in nonequilibrium properties of superconductors. A comprehensive review of recent developments in this field can be found in [40].

These yet unresolved issues, as well as some shortcomings of the models presented above, motivate the present work. We continue here our previous investigation on the phase diagrams of a BCS superconductor perturbed by a reduced $s$ - $d$ interaction [41] to examine the critical magnetic field and the effect of an external magnetic field $\mathcal{H}$ on the superconducting transition temperature $T_{\mathrm{c}}$ of such system. To this end we use the following model Hamiltonian

$$
H^{(M)}=H_{\mathrm{BCS}}+V^{(M)}
$$

where

$$
H_{\mathrm{BCS}}=H_{0}+V_{\mathrm{BCS}}
$$

and

$$
H_{0}=\sum_{\mathbf{k} \sigma} \xi_{\mathbf{k}} n_{\mathbf{k} \sigma}
$$

with $\xi_{\mathbf{k}}=\varepsilon_{\mathbf{k}}-\mu, n_{\mathbf{k} \sigma}=a_{\mathbf{k} \sigma}^{\dagger} a_{\mathbf{k} \sigma}$ and $\sigma= \pm$ denoting spin, is the free fermion kinetic energy operator and

$$
V_{\mathrm{BCS}}=-|\Lambda|^{-1} \sum_{\mathbf{k k}^{\prime}} G_{\mathbf{k}^{\prime}} a_{\mathbf{k}+}^{\dagger} a_{-\mathbf{k}-}^{\dagger} a_{-\mathbf{k}^{\prime}-} a_{\mathbf{k}^{\prime}+},
$$

is the Cooper pairing potential, whereas

$$
V^{(M)}=g^{2} N^{-1} \sum_{\alpha=1}^{M} \sigma_{z} S_{z \alpha}
$$

represents the reduced $s$ - $d$ interaction. $|\Lambda|$ denotes the system's volume and $G_{\mathbf{k}^{\prime}}$ is real, symmetric, invariant under $\mathbf{k} \rightarrow-\mathbf{k}$ or $\mathbf{k}^{\prime} \rightarrow-\mathbf{k}^{\prime}$ and nonvanishing only in a thin shell around the Fermi surface, viz.,

$$
G_{\mathbf{k k}^{\prime}}=G_{0} \chi(\mathbf{k}) \chi\left(\mathbf{k}^{\prime}\right), \quad G_{0}>0
$$

where $\chi(\mathbf{k})$ denotes the characteristic function of the set

$$
\mathcal{P}=\left\{\mathbf{k}: \mu-\delta \leq \varepsilon_{\mathbf{k}} \leq \mu+\delta\right\}, \quad \varepsilon_{\mathbf{k}}=\frac{\hbar^{2} \mathbf{k}^{2}}{2 m}
$$


In equation (5), $S_{z \alpha}$ denotes the spin operator of the $\alpha$ th magnetic ion, whereas

$$
\sigma_{z}=\sum_{\mathbf{k} \in \mathcal{P}}\left(n_{\mathbf{k}+}-n_{\mathbf{k}-}\right)
$$

is the spin operator of a conducting fermion. $M$ is the number of magnetic impurities, $N$ the number of host atoms.

We assume the perturbation implemented by the localized distinguishable magnetic impurities to be a reduced long-range $s$ - $d$ interaction, which involves only the $z$-components of the impurity and fermion spin operators (5). The reason for this simplification is that the thermodynamics of the resulting Hamiltonian $H^{(M)}=$ $H_{0}+V_{\mathrm{BCS}}+V^{(M)}$ admits a mean-field solution, the accuracy of which improves with decreasing impurity density.

In our approach the impurity effects depend on the magnetic coupling constant $g$ and impurity concentration $c=M N^{-1}$. However, it does not include the phase function, which appears in the Green's function method $[31,42]$.

In Section 2 the system's free energy $F(H, \beta)$ is determined by making use of the Bogolyubov inequality [43]. The resulting approximate expression for the free energy is employed in Sections 3 and 4 to derive the equation for the critical magnetic field $\mathcal{H}_{\mathrm{c}}$. The credibility of the theoretical expression for $\mathcal{H}_{\mathrm{c}}$, which depends on the magnetic coupling constant $g$ and impurity concentration $c$, is subsequently verified on the experimental data for LaCe, ThGd and $\mathrm{SmRh}_{4} \mathrm{~B}_{4}$. Good quantitative agreement with experiment was obtained. At low temperatures, the critical magnetic field is found to increase with decreasing temperature, similarly as in some antiferromagnetic superconductors.

Section 5 is devoted to study of the effect of an external magnetic field on superconducting alloys. Results of this study are exploited in Section 6 to derive the expression for $T_{\mathrm{c}}(\mathcal{H})$.

The theory presented improves earlier developments in this field. Apart from an explanation of the reentrant behaviour of superconducting alloys [41], it clarifies the Jaccarino-Peter effect and provides good quantitative agreement with experimental data of several superconducting alloys, i.e. $\mathrm{LaCe}$, ThGd and $\mathrm{SmRh}_{4} \mathrm{~B}_{4}$.

\section{Upper bound to the free energy in terms of the Bogolyubov method}

The full Hamiltonian of the system

$$
H^{(M)}=H_{0}+V_{\mathrm{BCS}}+V^{(M)}
$$

can be expressed in the following form in terms of meanfield parameters $\nu, \eta$ :

$$
H^{(M)}=h^{(M)}(\nu, \eta)+H_{R}^{(M)}
$$

where

$$
\begin{aligned}
h^{(M)}(\nu, \eta) & =\tilde{h}+h_{i m p}^{(M)}+\frac{1}{2} M N\left(\nu^{2}-\eta^{2}\right), \\
\tilde{h} & =H_{\mathrm{BCS}}+\kappa \sigma_{z}, \quad \kappa=-g M(\nu-\eta) \\
h_{i m p}^{(M)} & =g \nu \sum_{\alpha} S_{z \alpha}+\frac{1}{2} N^{-1} g^{2} \sum_{\alpha} S_{z \alpha}^{2}, \\
H_{R}^{(M)} & =-\frac{1}{2} N^{-1} \sum_{\alpha}\left[g\left(\sigma_{z}-S_{z \alpha}\right)-\nu N\right]^{2} \\
& +\frac{1}{2} N^{-1} \sum_{\alpha}\left(g \sigma_{z}-\eta N\right)^{2} .
\end{aligned}
$$

The Bogolyubov inequality [43]

$$
\left.\left.F\left(H_{1}+H_{2}\right) \leq F\left(H_{1}\right)+\right\rangle H_{2}\right\rangle_{H_{1}},
$$

with $H_{1}=h^{(M)}(\nu, \eta)$, yields

$$
F\left(H^{(M)}, \beta\right) \leq F\left(h^{(M)}(\nu, \eta), \beta\right)+\left\langle H^{(R)}\right\rangle_{h^{(M)}},
$$

where

$$
\langle A\rangle_{B}=\frac{\operatorname{Tr} A \exp [-\beta B]}{\operatorname{Tr} \exp [-\beta B]} .
$$

The parameters $\nu$ and $\eta$ will be now chosen so that they minimize the free energy

$$
F\left(h^{(M)}(\nu, \eta), \beta\right)=-\beta^{-1} \ln \operatorname{Tr} \exp \left[-\beta h^{(M)}(\nu, \eta)\right],
$$

viz.,

$$
\frac{\partial F\left(h^{(M)}(\nu, \eta), \beta\right)}{\partial \nu}=0, \quad \frac{\partial F\left(h^{(M)}(\nu, \eta), \beta\right)}{\partial \eta}=0 .
$$

The explicit form of these equations is

$$
\begin{aligned}
\nu & =\frac{g}{N}\left\langle\sigma_{z}\right\rangle_{\tilde{h}}-\frac{g}{N}\left\langle S_{z}\right\rangle_{h_{i m p}^{(1)}}, \\
\eta & =\frac{g}{N}\left\langle\sigma_{z}\right\rangle_{\tilde{h}} .
\end{aligned}
$$

Using equations (11), (14) and (15) one obtains

$$
\left\langle H^{(R)}\right\rangle_{h^{(M)}}=-\frac{1}{2} c g^{2}\left(\left\langle S_{z}^{2}\right\rangle_{h_{i m p}^{(1)}}-\left\langle S_{z}\right\rangle_{h_{i m p}^{(1)}}^{2}\right) .
$$

The inequality $\operatorname{Tr}\left(\rho A^{2}\right) \geq(\operatorname{Tr}(\rho A))^{2}$, valid for any bounded self-adjoint operator $A$ and density matrix $\rho$, shows that $\left\langle H^{(R)}\right\rangle_{h^{(M)}} \leq 0$. Hence, from equations (13), (16) one obtains the relevant upper bound to the free energy

$$
F\left(H^{(M)}, \beta\right) \leq F\left(h^{(M)}(\nu, \eta), \beta\right) .
$$

According to equation (17) we ascertain that the thermodynamics of the original system, characterized by $H^{(M)}$, is almost equivalent to that of $h^{(M)}$, provided $\eta$ and $\nu$ are the minimizing solutions of equations (14) and (15). 
The consequences of disregarding a term $\left\langle H^{(R)}\right\rangle_{h^{(M)}}$ are discussed in Section 4.

The two equations (14) and (15) can be reduced to a single one for $\nu$. The only requirement is $g>0$. The general form of equations (14) and (15) is

$$
\begin{aligned}
& \nu=f_{1}(\nu-\eta)+f_{2}(\nu), \\
& \eta=f_{1}(\nu-\eta) .
\end{aligned}
$$

Let $g>0$, then $f_{2}>0$. Furthermore,

$$
\eta=\nu-f_{2}(\nu)
$$

which yields the equation for $\nu$ :

$$
\nu=f_{1}\left(f_{2}(\nu)\right)+f_{2}(\nu)
$$

where according to equations (18) and (19):

$$
\begin{aligned}
& f_{1}(\nu)=(M N \beta)^{-1} \frac{\partial}{\partial \nu} \ln \operatorname{Tr} \exp [-\beta \tilde{h}(\nu, 0)] \\
& f_{2}(\nu)=(N \beta)^{-1} \frac{\partial}{\partial \nu} \ln \operatorname{Tr} \exp \left[-\beta h_{i m p}^{(1)}\right] .
\end{aligned}
$$

\section{Mean-field theory of $\tilde{h}$}

The form of the Hamiltonian $\tilde{h}$, given by equation (9) is analogous to the following Hamiltonian:

$$
H_{\mathrm{BCS}}(\mathcal{H})=H_{0}+V_{\mathrm{BCS}}-\mu_{\mathrm{B}} \mathcal{H} \sigma_{z}
$$

describing a system of electrons with attractive BCS interaction in the presence of an external magnetic field $\mathcal{H}$ ( $\mu_{\mathrm{B}}$ denotes the Bohr magneton). The explicit form of the system's free energy $F\left(h^{(M)}(\nu, \eta), \beta\right)$ can be therefore derived by exploiting the Bogolyubov-Valatin transformation $[44,45]$ and the method developed in [46] for $H_{\mathrm{BCS}}(\mathcal{H})$.

This has been done in [41] for spin $S=1 / 2$ and $S=7 / 2$ magnetic impurities perturbing the BCSsuperconductor. For further investigation, let us recall the final form of the free energy $F^{(S)}$ and briefly recapitulate the results of [41].

The free energy is given by the following equation:

$$
\begin{aligned}
F^{(S)}= & \min _{\{\Delta, \nu\}}\left\{\rho _ { F } | \Lambda | \int _ { - \delta } ^ { \delta } \left[\frac{1}{2} \Delta^{2} E^{-1} f_{3}\left(\beta, E, \xi, f_{2}^{(S)}\right)\right.\right. \\
& \left.-\beta^{-1} \ln \left[2 \cosh (\beta E)+2 \cosh \left(g \beta M f_{2}^{(S)}\right)\right]\right] d \xi \\
& +M^{2} c^{-1}\left(\nu f_{2}^{(S)}-\frac{1}{2}\left(f_{2}^{(S)}\right)^{2}\right) \\
& \left.+F_{i m p}^{(S)}+E_{0}(\Delta=0)+\rho_{F} \delta^{2}\right\}, \quad S=1 / 2,7 / 2,
\end{aligned}
$$

where $F_{i m p}^{(S)}$ is the free energy of impurity subsystem, given by (32) and (34), $E=\sqrt{\Delta^{2}+\xi^{2}}, \rho_{F}$ denoting the density of states at Fermi level,

$$
\rho_{F}=\frac{m p_{F}}{2 \pi \hbar^{2}}
$$

whereas $E_{0}(\Delta=0)$ denotes the ground state energy of free fermions. Two last terms in (25) are the contribution to the free energy density from one-fermion states, lying outside $\mathcal{P}$.

The system's state is characterized, according to equation (25), by the minimizing solution, $\left\{\Delta_{\mathrm{m}}, \nu_{\mathrm{m}}\right\}$, of the following set of equations for the gap $\Delta$ and a parameter $\nu$, describing the impurity subsystem

$$
\Delta=\frac{1}{2} G_{0} \rho \int_{-\delta}^{\delta} \frac{\Delta}{E} f_{3}\left(\beta, E, \xi, f_{2}^{(S)}(\nu)\right) d \xi \quad S=1 / 2,7 / 2,
$$

$$
\begin{aligned}
\nu & =f_{1}\left(f_{2}^{(S)}(\nu)\right)+f_{2}^{(S)}(\nu) \\
& =\frac{c g}{M} \frac{\sinh \left(\beta g M f_{2}^{(S)}(\nu)\right)}{\cosh \left(\beta g M f_{2}^{(S)}(\nu)\right)+\cosh \left(\beta E_{\mathbf{k}}\right)}+f_{2}^{(S)}(\nu),
\end{aligned}
$$

where

$$
f_{3}\left(\beta, E, \xi, f_{2}\right)=\frac{\sinh (\beta E)}{\cosh (\beta E)+\cosh \left(g \beta M f_{2}^{(S)}(\nu)\right)} .
$$

The properties of a superconductor with magnetic impurities can be determined by solving this set of equations, which is supplemented by the following condition for the chemical potential $\mu$ :

$$
\sum_{\mathbf{k} \sigma} \operatorname{Tr}\left(n_{\mathbf{k} \sigma} \rho_{0}\right)=n
$$

where $n_{\mathbf{k} \sigma}=a_{\mathbf{k} \sigma}^{\dagger} a_{\mathbf{k} \sigma}$ is the fermion number operator and $n$ denotes the average number of fermions in the system. It has been shown in [41], that this condition, takes the form:

$$
\sum_{\mathbf{k}}\left[1-\frac{\xi_{\mathbf{k}}}{E_{\mathbf{k}}} f_{3}\left(\beta, E_{\mathbf{k}}, \xi_{\mathbf{k}}, f_{2}^{(S)}\right)\right]=n
$$

Equation (30) resembles the BCS equation for $\mu$ and the properties of $f_{3}$ are similar to those of $f_{\mathrm{BCS}}=$ $\tanh \left(\beta E_{\mathbf{k}} / 2\right)$, e.g. both functions are odd in $\xi_{\mathbf{k}}$. The solution of (30) is therefore exactly the same as in BCS theory, viz., $\mu=\varepsilon_{\mathrm{F}}$. Thus, we assume that in the low-temperature scale the following relations hold:

$$
\mu=\varepsilon_{\mathrm{F}}, \quad \frac{\partial \mu}{\partial T}=0, \quad \rho=\rho_{\mathrm{F}} .
$$

Equations (26) and (27) clearly possess the solution $\Delta=$ $\nu=0$ for all values of $\beta \geq 0$. At sufficiently large values of $\beta$ one finds also other solutions, viz., $\{\Delta \neq 0, \nu=0\},\{\Delta=$ $0, \nu \neq 0\},\{\Delta \neq 0, \nu \neq 0\}$. Accordingly, we distinguish the following phases: 
- paramagnetic phase $P$ with $\left\{\Delta_{\mathrm{m}}=0, \nu_{\mathrm{m}}=0\right\}$,

- unperturbed superconducting state $S C$ with $\left\{\Delta_{\mathrm{m}} \neq 0\right.$, $\left.\nu_{\mathrm{m}}=0\right\}$,

- ferromagnetic phase $F$ without bound Cooper pairs and $\left\{\Delta_{\mathrm{m}}=0, \nu_{\mathrm{m}} \neq 0\right\}$, in which impurity spins tend to align opposite to those of conduction fermions (cf. Eqs. (9) and (10)),

- intermediate phase $D$ in which superconductivity coexists with ferromagnetism and $\left\{\Delta_{\mathrm{m}} \neq 0, \nu_{\mathrm{m}} \neq 0\right\}$.

We define the following temperatures corresponding to the respective phase transitions

- $T_{\mathrm{c}}$, 2nd order transition $S C \rightarrow P$,

- $T_{P F}$, Curie temperature of 2nd order transition $F \rightarrow P$,

- $T_{S C D}, 1$ st order transition $D \rightarrow S C$,

- $T_{F D}, 1$ st order transition $D \rightarrow F$,

- $T_{S C F}, 1$ st order transition $S C \rightarrow F$.

The set of equations (26) and (27) is solved numerically and the results are presented in Section 4.

The free energy (25), as well as the equations for $\Delta(26)$ and $\nu(27)$ strongly depend on the value of the impurity spin $S$. In the present work we study the influence on superconductivity of the following magnetic ions: Ce $(S=$ $1 / 2)$, Gd and $\operatorname{Sm}(S=7 / 2)$. For $S=1 / 2$ one obtains

$$
\begin{aligned}
F_{i m p}^{\left(\frac{1}{2}\right)} & =-\beta^{-1} \ln \operatorname{Tr} \exp \left[-\beta h_{i m p}^{(M)}\right] \\
& =-\beta^{-1} \sum_{\alpha=1}^{M} \ln \operatorname{Tr} \exp \left[-\beta h_{i m p}^{(1)}\right] \\
& =-M \beta^{-1} \ln [2 \cosh (\beta g \nu)]+\frac{1}{2} c g^{2},
\end{aligned}
$$

with

$$
f_{2}^{\left(\frac{1}{2}\right)}(\nu)=\frac{c g}{M} \tanh (\beta g \nu) .
$$

Accordingly, for spin $7 / 2$ impurities

$$
\begin{aligned}
F_{i m p}^{\left(\frac{7}{2}\right)}= & -M \beta^{-1} \ln 2\left[\exp \left[-24 g^{2} \beta N^{-1}\right] \cosh (7 \beta g \nu)\right. \\
& +\exp \left[-12 g^{2} \beta N^{-1}\right] \cosh (5 \beta g \nu) \\
& \left.+\exp \left[-4 g^{2} \beta N^{-1}\right] \cosh (3 \beta g \nu)+\cosh (\beta g \nu)\right] \\
& +\frac{1}{2} c g^{2},
\end{aligned}
$$

where

$$
\begin{aligned}
f_{2}^{\left(\frac{7}{2}\right)}(\nu)= & \frac{c g}{M R}\left[7 \exp \left[-24 g^{2} \beta N^{-1}\right] \sinh (7 \beta g \nu)\right. \\
& +5 \exp \left[-12 g^{2} \beta N^{-1}\right] \sinh (5 \beta g \nu) \\
+ & \left.3 \exp \left[-4 g^{2} \beta N^{-1}\right] \sinh (3 \beta g \nu)+\sinh (\beta g \nu)\right],
\end{aligned}
$$

and

$$
\begin{aligned}
R= & \exp \left[-24 g^{2} \beta N^{-1}\right] \cosh (7 \beta g \nu) \\
& +\exp \left[-12 g^{2} \beta N^{-1}\right] \cosh (5 \beta g \nu) \\
& +\exp \left[-4 g^{2} \beta N^{-1}\right] \cosh (3 \beta g \nu)+\cosh (\beta g \nu) .
\end{aligned}
$$

The complexity of the expressions for the free energy $F^{(S)}$ and functions $f_{1}, f_{2}, f_{3}$ increases with the impurity spin value. It follows, that the impurity spin is the key factor affecting the thermodynamics of superconducting magnetic alloys. This conclusion is complementary with the fundamental experimental observation made by Matthias et al. [6].

\section{The critical magnetic field}

The critical magnetic field $\mathcal{H}_{\mathrm{c} \Phi}$ forcing a system to undergo the phase transition from the $\Phi$ phase to paramagnetic (normal) phase $(P)$ is given by the equation

$$
H_{\mathrm{c} \Phi}^{2}=\left(F_{P}-F_{\Phi}\right) / 2 \mu_{0},
$$

where $\mu_{0}$ denotes the vacuum permeability, $F_{P}$ and $F_{\Phi}$ denote the free energy of the $P$ and $\Phi$ phase, respectively.

The free energy of the normal state can be obtained from (25) with $\Delta=0$ and $\nu=0$, which yields:

- for spin $1 / 2$ impurities

$$
\begin{aligned}
F_{P}^{\left(\frac{1}{2}\right)}= & -2 \rho_{F}|\Lambda| \beta^{-1} \int_{-\delta}^{\delta} \ln 2 \cosh \left(\frac{1}{2} \beta \xi\right) d \xi \\
& -M \beta^{-1} \ln 2+\frac{1}{2} c g^{2}+E_{0}(\Delta=0)+\rho_{F} \delta^{2},
\end{aligned}
$$

- for spin $7 / 2$ impurities

$$
\begin{aligned}
F_{P}^{\left(\frac{7}{2}\right)}= & -2 \rho_{F}|\Lambda| \int_{-\delta}^{\delta} \ln 2 \cosh \left(\frac{1}{2} \beta \xi\right) d \xi \\
& -M \beta^{-1} \ln 2\left[\exp \left[-24 \beta c g^{2} M^{-1}\right]\right. \\
& -\exp \left[-12 \beta c g^{2} M^{-1}\right] \\
& \left.-\exp \left[-4 \beta c g^{2} M^{-1}\right]+1\right]+\frac{1}{2} c g^{2} \\
& +E_{0}(\Delta=0)+\rho_{F} \delta^{2}
\end{aligned}
$$

The critical magnetic field, which completely suppresses superconductivity, (i.e. induces a phase transition $S C \rightarrow$ $P$ ) is given by (36) with $F_{S C}$ replacing $F_{\Phi}$. It will be denoted as usual by $\mathcal{H}_{\mathrm{c}}$. The expression for $F_{S C}$ results from (25) with $\{\Delta \neq 0, \nu=0\}$ :

$$
\begin{aligned}
F_{S C}^{\left(\frac{1}{2}\right)}= & \rho_{F}|\Lambda| \int_{-\delta}^{\delta}\left[\frac{1}{2} \Delta^{2} E^{-1} f_{3}^{(B C S)}(\beta, E, \xi)\right. \\
& \left.-2 \beta^{-1} \ln 2 \cosh (\beta E)\right] d \xi \\
& +\frac{1}{2} c g^{2}+E_{0}(\Delta=0)+\rho_{F} \delta^{2}, \quad \text { for } S=1 / 2,
\end{aligned}
$$




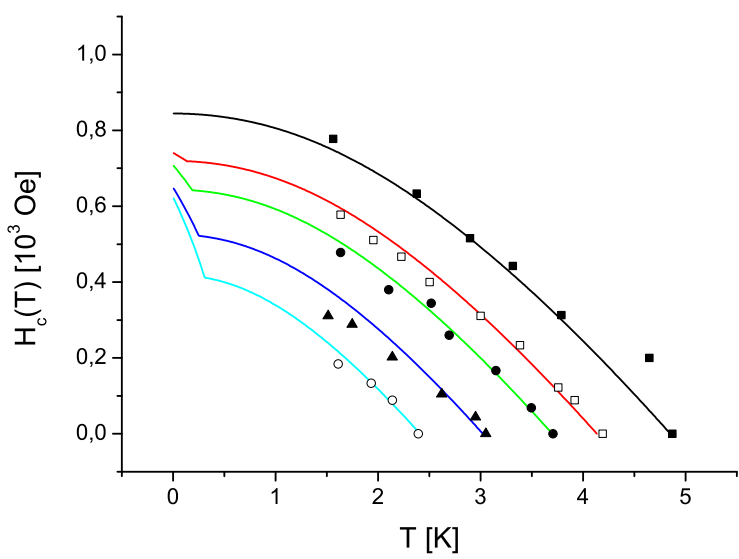

(a)

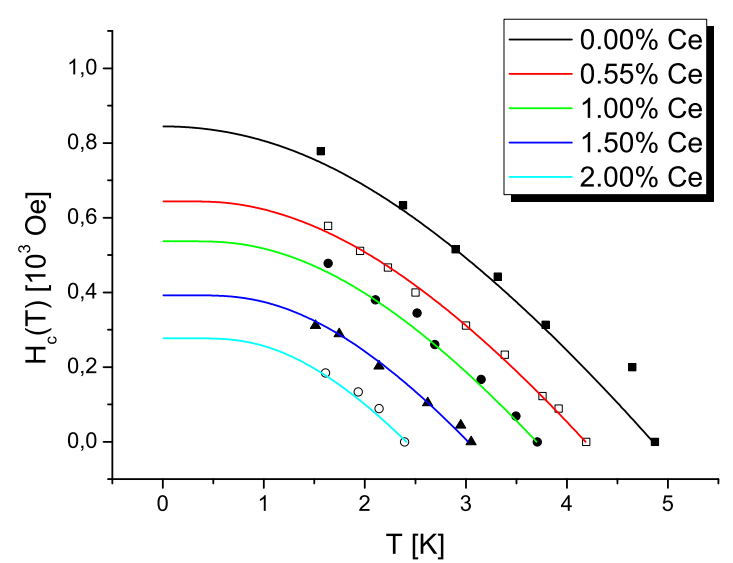

(b)

Fig. 1. The critical magnetic field of LaCe under varying Ce concentration for real temperature $T$ and parameter values given in Table 1 (a) and for the effective temperature $\tilde{T}$ and parameter values given in Table 2 (b). The points are experimental data from $[47]$.

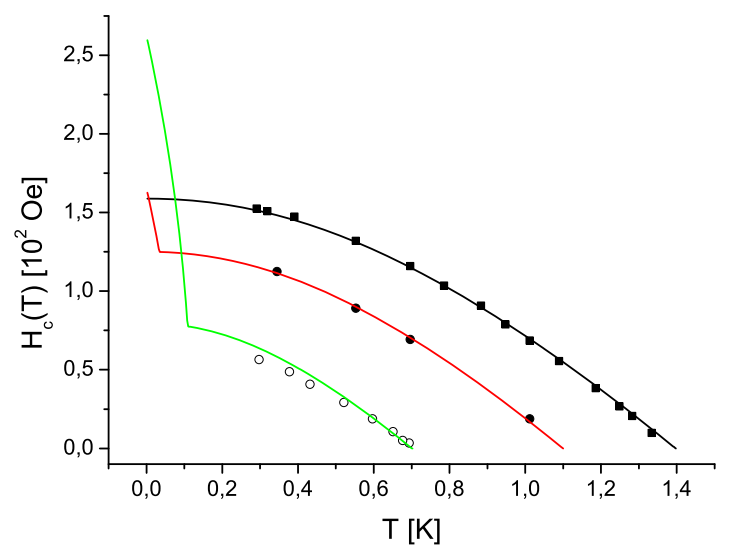

(a)

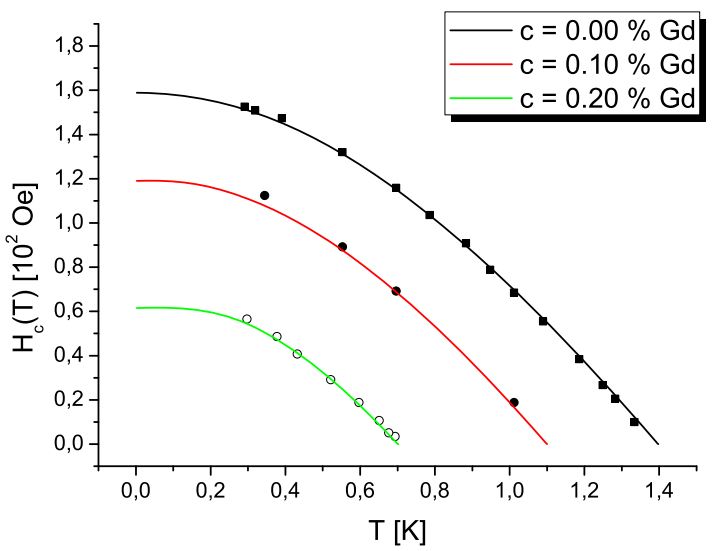

(b)

Fig. 2. The critical magnetic field of ThGd under varying Gd concentration for: real temperature $T$ and parameter values given in Table 1 (a) the effective temperature $\tilde{T}$ and parameter values given in Table 2 (b). The points are experimental data from [48].

and

$$
\begin{aligned}
F_{S C}^{\left(\frac{7}{2}\right)}= & \rho_{F}|\Lambda| \int_{-\delta}^{\delta}\left[\frac{1}{2} \Delta^{2} E^{-1} f_{3}^{(B C S)}(\beta, E, \xi)\right. \\
& \left.-2 \beta^{-1} \ln 2 \cosh (\beta E)\right] d \xi \\
& -M \beta^{-1} \ln 2\left[\exp \left[-24 \beta c g^{2} M^{-1}\right]\right. \\
& \left.-\exp \left[-12 \beta c g^{2} M^{-1}\right]-\exp \left[-4 \beta c g^{2} M^{-1}\right]+1\right] \\
& +\frac{1}{2} c g^{2}+E_{0}(\Delta=0)+\rho_{F} \delta^{2}, \quad \text { for } S=7 / 2 .
\end{aligned}
$$

The credibility of the above theoretical expressions will be now verified on the experimental data of LaCe, ThGd and $\mathrm{SmRh}_{4} \mathrm{~B}_{4}$. The parameters $g, G_{0} \rho_{F}, \delta, M$ were adjusted to fit the experimental data. In order to perform this fit, the set of equations (26) and (27) was solved numerically for fixed values of the parameters $g, \rho_{F}|\Lambda|, G_{0} \rho_{F}, \delta, M$.
The minimizing solution of this set of equations was subsequently substituted to the expressions for $\mathcal{H}_{\mathrm{c}}$ or $\mathcal{H}_{\mathrm{c} D}$ depending on which of the system's states $(S C$ or $D)$ possess the smaller values of the free energy.

The critical magnetic field of LaCe, ThGd and $\mathrm{SmRh}_{4} \mathrm{~B}_{4}$ are depicted in Figures 1a, 2a and 3, respectively. The sample solutions of the set of equations for $\Delta$ and $\nu$ under varying temperature and impurity concentration of $\mathrm{Ce}$ and $\mathrm{Gd}$ are depicted in Figures 4-7. The agreement of theoretical and experimental data is satisfactory. The discrepancies, which increase with decreasing temperature and increasing impurity concentration in case of LaCe and ThGd, can be reduced by introducing the concept of the effective inverse temperature $\tilde{\beta}=1 / k_{\mathrm{B}} \tilde{T}$. The latter is related to the system's real temperature by the following expression

$$
\tilde{\beta}(\beta, \gamma)=\gamma^{-1} \tanh (\beta \gamma)
$$




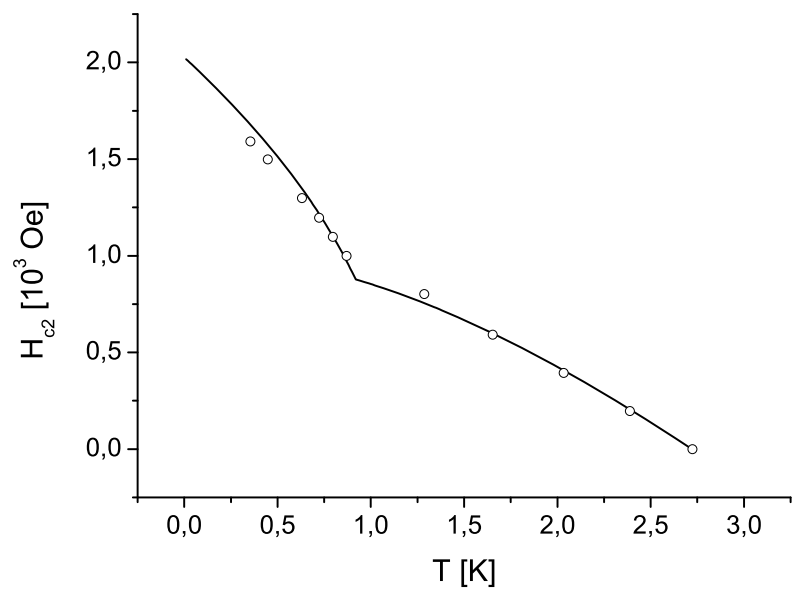

Fig. 3. The upper critical magnetic field $\mathcal{H}_{\mathrm{c} 2}$ of $\mathrm{SmRh}_{4} \mathrm{~B}_{4}$ for the parameter values given in Table 1 . The points are experimental data from [36]. $\mathcal{H}_{\mathrm{c} 2}$ is related to the thermodynamic critical magnetic field $\mathcal{H}_{\mathrm{c}}$ by the expression, $\mathcal{H}_{\mathrm{c} 2}=\kappa \sqrt{2} H_{\mathrm{c}}$. The Ginzburg-Landau parameter $\kappa$ has been treated as the additional adjustable parameter used to fit experimental data and assumed to be independent in $T$. The value of $\kappa$ providing best fit is $\kappa=1.51$.

Table 1. The parameter values exploited during the numerical analysis of equations (26), (27) and (36).

\begin{tabular}{cccccc}
\hline Alloy & $c[\%]$ & $M$ & $g[\sqrt{\mathrm{eV}}]$ & $\delta[\mathrm{eV}]$ & $G_{0} \rho_{F}$ \\
\hline LaCe & 0.00 & 0 & 0.000 & 0.01 & 0.3030 \\
LaCe & 0.55 & 2 & 0.080 & 0.01 & 0.2890 \\
LaCe & 1.00 & 4 & 0.095 & 0.01 & 0.2800 \\
LaCe & 1.50 & 5 & 0.100 & 0.01 & 0.2650 \\
LaCe & 2.00 & 6 & 0.105 & 0.01 & 0.2500 \\
\hline ThGd & 0.00 & 0 & 0.00 & 0.01 & 0.2200 \\
ThGd & 0.10 & 3 & 0.11 & 0.01 & 0.2090 \\
ThGd & 0.20 & 5 & 0.18 & 0.01 & 0.1910 \\
\hline $\mathrm{SmRh}_{4} \mathrm{~B}_{4}$ & 11.11 & 7 & 0.0625 & 0.0106 & 0.2540 \\
\hline
\end{tabular}

The effective temperature results by averaging (over impurity positions) the single particle equilibrium density matrix of a quantum particle in a field of randomly positioned wells, representing the screened Coulomb potential at each impurity site [49]. Furthermore, it has been shown, that $\gamma$ is of the form

$$
\gamma=\frac{\hbar}{2} \sqrt{M u_{2} m^{-1}}
$$

with $u_{2}$ denoting the 2 nd derivative at well's minimum. In the present work $\gamma$ will be treated as the adjustable parameter.

Theoretical curves of the critical magnetic field of LaCe and ThGd superconducting alloys with $\tilde{\beta}$ replacing $\beta$ in (36) are given in Figures $1 \mathrm{~b}$ and $2 \mathrm{~b}$. It is clear, that the application of the effective temperature improves the agreement of the given model with experimental data for LaCe and ThGd, proving that the Coulomb interactions (apart from exchange interactions) between impurity ions and conduction fermions shall be also taken into account in development of superconducting alloys theory.
Table 2. The parameter values exploited during the numerical analysis of equations (26), (27) and (36).

\begin{tabular}{ccccccc}
\hline Alloy & $c[\%]$ & $M$ & $g[\sqrt{\mathrm{eV}}]$ & $\delta[\mathrm{eV}]$ & $G_{0} \rho_{F}$ & $\gamma\left[10^{-4} \mathrm{eV}\right]$ \\
\hline LaCe & 0.00 & 0 & 0.000 & 0.01 & 0.3030 & 0.00 \\
LaCe & 0.55 & 2 & 0.080 & 0.01 & 0.2900 & 1.20 \\
LaCe & 1.00 & 4 & 0.095 & 0.01 & 0.2810 & 1.30 \\
LaCe & 1.50 & 5 & 0.100 & 0.01 & 0.2655 & 1.50 \\
LaCe & 2.00 & 6 & 0.105 & 0.01 & 0.2520 & 1.50 \\
\hline ThGd & 0.00 & 0 & 0.00 & 0.01 & 0.2200 & 0.00 \\
ThGd & 0.10 & 3 & 0.11 & 0.01 & 0.2090 & 0.20 \\
ThGd & 0.20 & 5 & 0.18 & 0.01 & 0.1915 & 0.30 \\
\hline
\end{tabular}

The effective temperature has been also proven to be the crucial in description of the doping dependence of superconducting transition temperature $T_{\mathrm{c}}$ in high- $T_{\mathrm{c}}$ superconductors [50].

In case of $\mathrm{SmRh}_{4} \mathrm{~B}_{4}$, the concentration of magnetic impurities $\left(\mathrm{Sm}^{3+}\right.$ ions), $c \approx 11 \%$ is much larger than in case of LaCe and ThGd. Accordingly, the agreement with experimental data for $\mathrm{SmRh}_{4} \mathrm{~B}_{4}$ is only satisfactory and cannot be improved by the application of the effective temperature. This suggests, that the term $\left\langle H^{(R)}\right\rangle_{h(M)}$ cannot be disregarded for sufficiently large $c$ and shall be included in the free energy computation or the dependence of the Ginzburg-Landau parameter $\kappa$ on $T$ shall be taken into account. In the case of large $c$, the magnitude of exchange interaction between conduction fermions and magnetic ions may presumably exceeds the magnitude of the Coulomb attraction between magnetic ions and conduction fermions.

The graphs, depicted in Figures 1a and 2a show an increase of the critical magnetic field at very low temperature scale and for sufficiently large values of impurity concentrations, e.g. for $c=2.0 \%$ Ce at Figure 1 a and for $c=0.20 \% \mathrm{Gd}$ at Figure 2 .

This enhancement of superconductivity also increases with impurity spin and impurity concentration, since at extremely low temperatures, the following relation

$$
\mathcal{H}_{\mathrm{cD}}(c=0.20 \% \mathrm{Gd})>\mathcal{H}_{\mathrm{cD}}(c=0.10 \% \mathrm{Gd})
$$

holds.

The above observation results from the following fact. If the solution $\{\Delta \neq 0, \nu \neq 0\}$ minimizes the free energy, then the values of $\mathcal{H}_{\mathrm{c} D}$ are larger than $\mathcal{H}_{\mathrm{c}}$. Accordingly, one obtains an increase of the critical magnetic field below $T_{S C D}$, since at this temperature, the system undergoes a phase transition $S C \rightarrow D$ and $\mathcal{H}_{\mathrm{c} D}$ becomes equal to $\mathcal{H}_{\mathrm{c}}$. This conclusion may be recognized as incompatible with physical intuition, suggesting that the external magnetic field should gain the perturbative effect of magnetic impurities. As a result $\mathcal{H}_{\mathrm{c} D}$ should possess smaller values than $\mathcal{H}_{\mathrm{c}}$.

An increase of the upper critical magnetic field $\mathcal{H}_{\mathrm{c} 2}=$ $\kappa \sqrt{2} \mathcal{H}_{\mathrm{c}}$ with $\kappa=\lambda / \xi$, denoting the Ginzburg-Landau parameter, has been observed in the following materials: $\mathrm{SmRh}_{4} \mathrm{~B}_{4}, \mathrm{GdMo}_{6} \mathrm{~S}_{8}, \mathrm{TbMo}_{6} \mathrm{~S}_{8}, \mathrm{Sn}_{1-x} \mathrm{Eu}_{x} \mathrm{Mo}_{6} \mathrm{~S}_{8}$, $\mathrm{Pb}_{1-x} \mathrm{Eu}_{x} \mathrm{Mo}_{6} \mathrm{~S}_{8}, \mathrm{La}_{1.2-x} \mathrm{Eu}_{x} \mathrm{Mo}_{6} \mathrm{~S}_{8}[9,10]$. These experiments confirm the validity of inequality (42). 


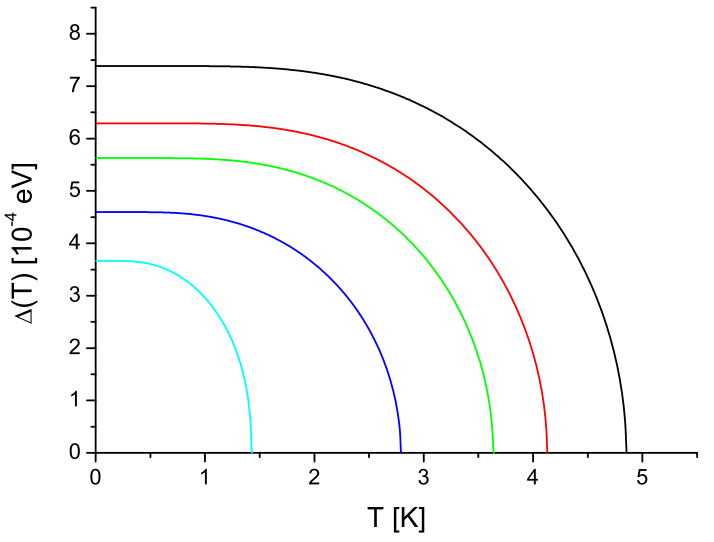

(a)

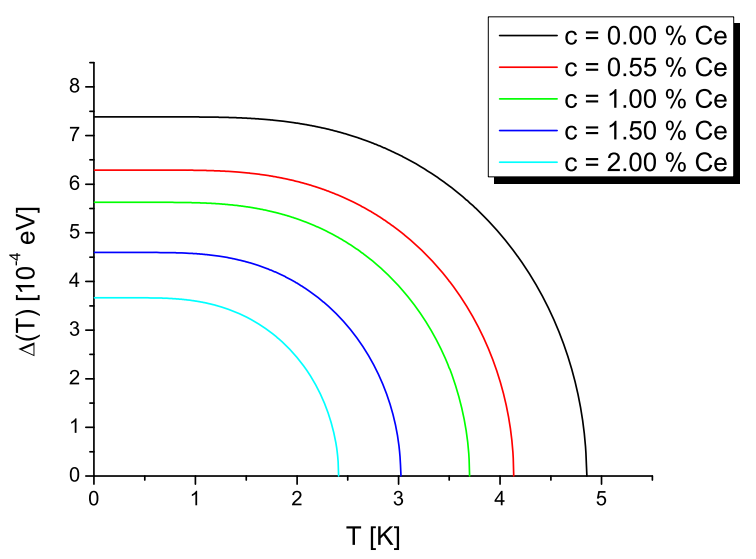

(b)

Fig. 4. The temperature dependence of the gap parameter $\Delta(T)$ for LaCe and the solution of equations (26) and (27) with $S=1 / 2$, in which $\left\{\Delta_{\mathrm{m}} \neq 0, \nu_{\mathrm{m}}=0\right\}$ (a) and $\{\Delta \neq 0, \nu \neq 0\}$ (b). The parameter values are given in Table 1 .

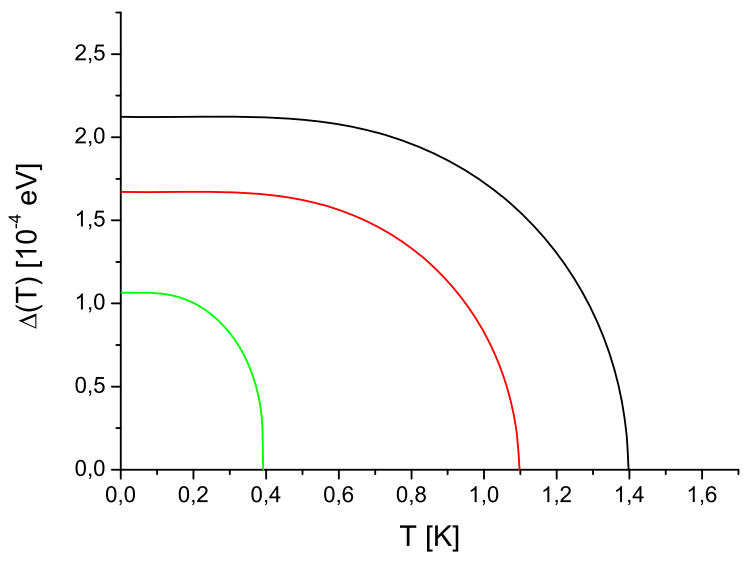

(a)

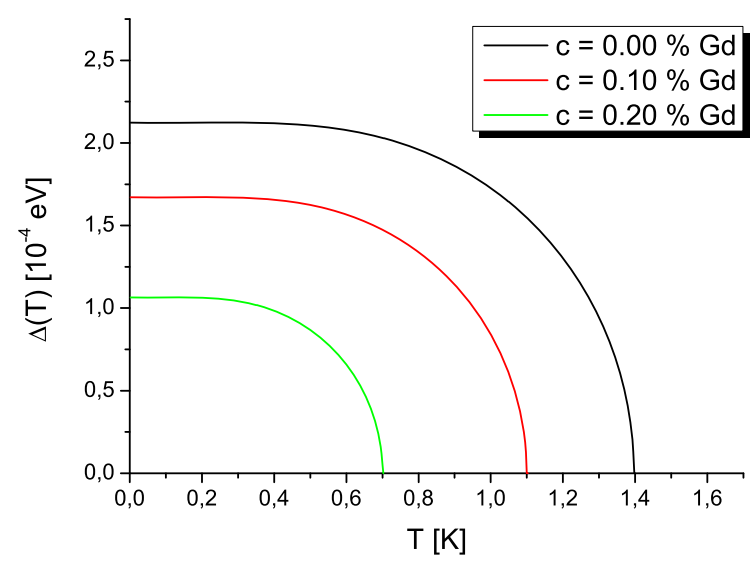

(b)

Fig. 5. The temperature dependence of the gap parameter $\Delta(T)$ for ThGd and the solution of equations (26) and (27) with $S=7 / 2$, in which $\left\{\Delta_{\mathrm{m}} \neq 0, \nu_{\mathrm{m}} \neq 0\right\}$ (a) and $\left\{\Delta_{\mathrm{m}} \neq 0, \nu_{\mathrm{m}}=0\right\}$ (b). The parameter values are given in Table 1 .

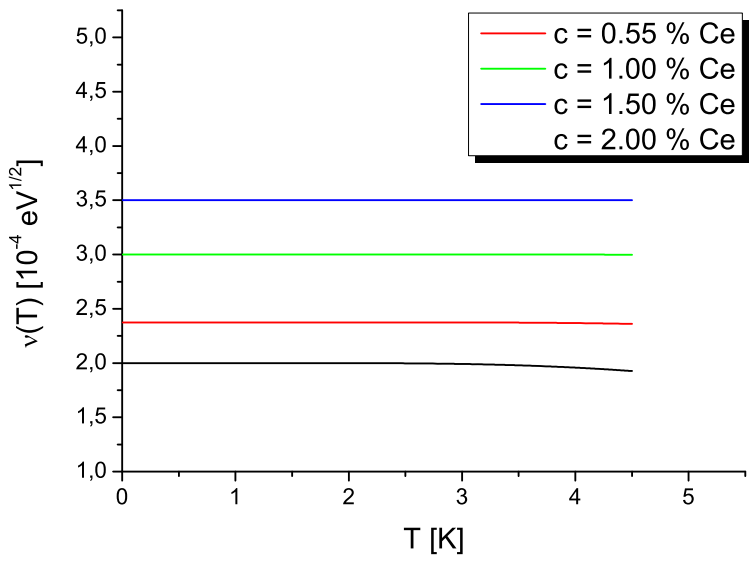

(a)

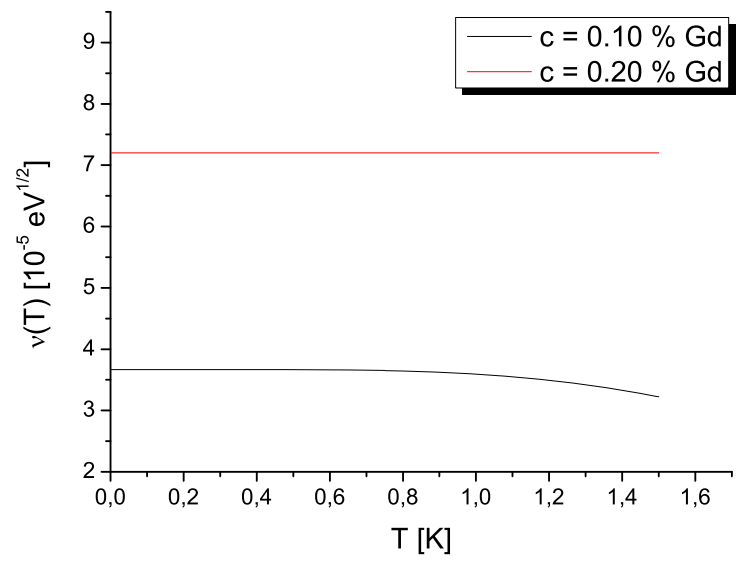

(b)

Fig. 6. The temperature dependence of the parameter $\nu(T)$ for the solution of equations (26) and (27), in which $\left\{\Delta_{\mathrm{m}} \neq 0, \nu_{\mathrm{m}} \neq 0\right\}$ for LaCe (a) and ThGd (b). The parameter values are given in Table 1. 


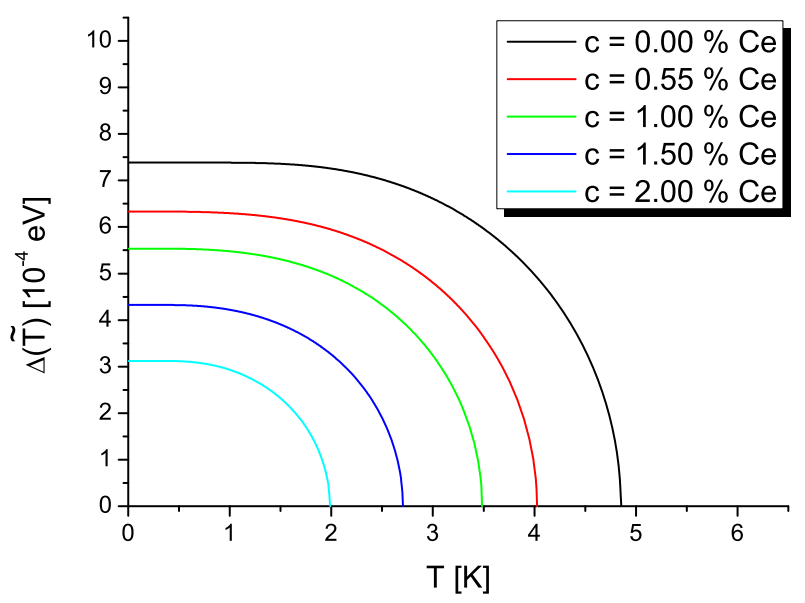

(a)

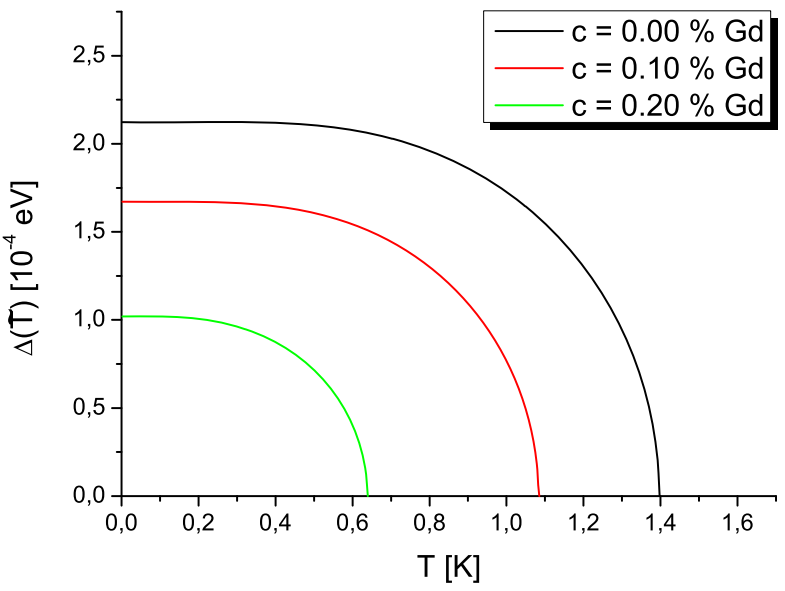

(b)

Fig. 7. The gap parameter $\Delta(\tilde{T})$ at the effective temperature $\tilde{T}$ and under varying impurity concentration for LaCe (a) and ThGd (b). The parameter values are given in Table 2 .

Fischer et al. [51] have pointed out that the superconductivity enhancement, represented by an increase of the critical magnetic field is a result of the JaccarinoPeter effect [24]. This phenomenon may occur in the IItype superconductor, in which the magnetic moments of the impurities are antiferromagnetically coupled to that of the conduction fermions. This interaction generates an exchange field $\mathcal{H}_{\mathrm{J}}$, which acts on the spins of conduction electrons equivalently to an applied magnetic field. Namely, breaks the Copper pairs. However, the negative sign of the coupling between the magnetic moments and the conduction fermions spins, determines the direction of $\mathcal{H}_{\mathrm{J}}$ to be opposite to that of $\mathcal{H}$. Thus, an applied magnetic field will be compensated by an exchange field, since the net magnetic field $\mathcal{H}_{\mathrm{T}}$ is given by $\mathcal{H}-\left|\mathcal{H}_{\mathrm{J}}\right|$. A given compound displays superconducting properties as long as the following relation holds

$$
-\mathcal{H}_{\mathrm{p}} \leq \mathcal{H}_{\mathrm{T}} \leq \mathcal{H}_{\mathrm{p}}
$$

where

$$
\mathcal{H}_{\mathrm{p}}=\sqrt{\frac{\rho_{F}}{\chi_{\mathrm{P}}-\chi_{\mathrm{SC}}}} .
$$

$\chi_{\mathrm{P}}$ and $\chi_{\mathrm{SC}}$ denote the magnetic susceptibility of the normal and superconducting state. $\mathcal{H}_{\mathrm{p}}$, defined by (44) is the Chandrasekhar-Clogston limiting paramagnetic field $[52,53]$.

The Jaccarino-Peter effect has been observed experimentally in $\mathrm{Eu}_{0.75} \mathrm{Sn}_{0.25} \mathrm{Mo}_{6} \mathrm{~S}_{7.2} \mathrm{Se}_{0.8}$ [25], during the investigation of the upper critical magnetic field. In particular, at low temperature scale, three subsequent phase transitions have been observed with increasing value of an external magnetic field, i.e. $S C \rightarrow P \rightarrow S C \rightarrow P$.

In conclusion, it is worth to point out, that the magnetic impurities were proven to limit the superconductivity, but on the other hand, under some specific conditions, they help the superconducting system to overcome the destructive effect of an external magnetic field. Furthermore, the interplay between superconductivity and magnetism is believed to be a possible mechanism of high- $T_{\mathrm{c}}$ superconductivity [54], since the undoped state of cuprate superconductors is a strongly insulating antiferromagnet. The existence of such a parent correlated insulator is viewed to be an essential feature of high temperature superconductivity.

In the above discussion we dealt only with the critical magnetic field. To fully judge, if the Jaccarino-Peter compensation may occur in the superconducting alloys, one should study the effect of an external magnetic field $\mathcal{H}$ on such system. This will be done in next two sections, in which we first study the effect of $\mathcal{H}$ on the superconducting alloys and then employ the resulting expressions to investigate the dependence of the superconducting critical temperature $T_{\mathrm{c}}$ on $\mathcal{H}$.

\section{The effect of an external magnetic field on the superconducting alloys}

In the presence of an external magnetic field $\mathcal{H}$, the system's Hamiltonian (6) should be supplemented by the additional terms, describing the interaction with a magnetic field. This yields [55]:

$$
H^{(M)}(\mathcal{H})=H^{(M)}-\frac{1}{2} g^{\prime} \mu_{\mathrm{B}} \tilde{\mathcal{H}} \sum_{\alpha} S_{z \alpha}-\mu_{\mathrm{B}} \mathcal{H} \sigma_{z},
$$

where $g^{\prime}$ is the modified Landé factor [56], $\mu_{\mathrm{B}}$ denotes the Bohr magneton, $\tilde{\mathcal{H}}=g_{0} \mathcal{H}$ is the effective magnetic field at each impurity site.

The additional electron $\sigma_{z}$ and impurity spin $S_{z}$ operators in (45) are separated. This allows to use thermodynamic equivalence of Hamiltonians $H^{(M)}$ and $h^{(M)}(\nu, \eta)$, proven in Section 2 to describe the thermodynamic properties of a BCS superconductor containing the magnetic 
impurities in the presence of magnetic field in terms of the Hamiltonian $h^{(M)}(\mathcal{H})$. The latter is of the following form:

$$
h^{(M)}(\mathcal{H})=h^{(M)}(\nu, \eta)-\frac{1}{2} g^{\prime} \mu_{\mathrm{B}} \tilde{\mathcal{H}} \sum_{\alpha} S_{z \alpha}-\mu_{\mathrm{B}} \mathcal{H} \sigma_{z}
$$

Accordingly, the electrons and impurities are described by the Hamiltonians $\tilde{h}$ and $h_{i m p}$, respectively,

$$
\begin{aligned}
\tilde{h} & =H_{\mathrm{BCS}}+\left(\kappa-\mu_{\mathrm{B}} \mathcal{H}\right) \sigma_{z} \\
& =H_{\mathrm{BCS}}-\left(g M f_{2}^{(S)}+\mu_{\mathrm{B}} \mathcal{H}\right) \sigma_{z} \\
& =H_{\mathrm{BCS}}-\lambda \sigma_{z}, \\
h_{i m p}(\mathcal{H}) & =g\left(\nu-\frac{1}{2 g} g^{\prime} \mu_{\mathrm{B}} \tilde{\mathcal{H}}\right) \sum_{\alpha} S_{z \alpha}+\frac{1}{2} N^{-1} g^{2} \sum_{\alpha} S_{z \alpha}^{2} \\
& =g \zeta \sum_{\alpha} S_{z \alpha}+\frac{1}{2} g^{2} N^{-1} \sum_{\alpha} S_{z \alpha}^{2} .
\end{aligned}
$$

The form of equations (46) and (48) is very similar to equations (9) and (10). It follows that, in order to include the effect of an external magnetic field on the free energy of a BCS superconductor containing magnetic impurities it suffices to perform the following substitutions in (25):

$$
\begin{aligned}
\kappa \rightarrow-\lambda & =\kappa-\mu_{\mathrm{B}} \mathcal{H}, \\
\nu \rightarrow \zeta & =\nu-\frac{1}{2 g} g^{\prime} \mu_{\mathrm{B}} \tilde{\mathcal{H}} .
\end{aligned}
$$

Accordingly, the set of equations for the parameters $\Delta(26)$ and $\nu(27)$ in the presence of the external magnetic field take the form:

$$
\begin{aligned}
\Delta & =\frac{1}{2} G_{0} \rho_{F} \int_{-\delta}^{\delta} \frac{\Delta}{E} f_{3}\left(\beta, E, \xi, f_{2}^{(S)}(\zeta), \mathcal{H}\right) d \xi \\
\nu & =f_{1}\left(\beta, E, \xi, f_{2}^{(S)}(\zeta), \mathcal{H}\right)+f_{2}^{(S)}(\zeta, \mathcal{H}), S=1 / 2,7 / 2,
\end{aligned}
$$

where

$$
\begin{aligned}
& f_{1}\left(\beta, E, \xi, f_{2}^{(S)}(\zeta), \mathcal{H}\right)= \\
& \quad \frac{c g}{M} \frac{\sinh \left[\beta\left(g M f_{2}^{(S)}(\zeta)+\mu_{\mathrm{B}} \mathcal{H}\right)\right]}{\cosh \left[\beta\left(g M f_{2}^{(S)}(\zeta)+\mu_{\mathrm{B}} \mathcal{H}\right)\right]+\cosh (\beta E)}, \\
& f_{3}\left(\beta, E, \xi, f_{2}^{(S)}(\zeta), \mathcal{H}\right)= \\
& \frac{\sinh (\beta E)}{\cosh (\beta E)+\cosh \left[\beta\left(g M f_{2}^{(S)}(\zeta)+\mu_{\mathrm{B}} \mathcal{H}\right)\right]} .
\end{aligned}
$$

Functions $f_{2}^{(S)}(\zeta)$ are given by equations (33) and (35), with $\zeta$ replacing $\nu$.

The free energy of the BCS superconductor perturbed by magnetic impurities and in the presence of an external magnetic field $\mathcal{H}$ then reads

$$
\begin{aligned}
F^{(S)}(\mathcal{H})= & \min _{\{\Delta, \nu\}}\left\{\rho _ { F } | \Lambda | \int _ { - \delta } ^ { \delta } \left[\frac{1}{2} \Delta^{2} E^{-1} f_{3}\right.\right. \\
& \times\left(\beta, E, \xi, f_{2}^{(S)}(\zeta), \mathcal{H}\right)-\beta^{-1} \ln [2 \cosh (\beta E) \\
& \left.\left.+2 \cosh \left[\beta\left(g M f_{2}^{(S)}(\zeta)+\mu_{\mathrm{B}} \mathcal{H}\right)\right]\right]\right] d \xi \\
& +M^{2} c^{-1}\left(\nu f_{2}^{(S)}(\zeta)-\frac{1}{2}\left(f_{2}^{(S)}(\zeta)\right)^{2}\right) \\
& \left.+F_{i m p}^{(S)}(\mathcal{H})+E_{0}(\Delta=0)+\rho_{F} \delta^{2}\right\} \\
S= & 1 / 2,7 / 2
\end{aligned}
$$

where $F_{i m p}^{(S)}(\mathcal{H})$ are given by equations (32) and (34) after substitution $\zeta \rightarrow \nu$.

\section{Critical temperature}

The phase diagrams of a BCS superconductor perturbed by magnetic impurities depicted by us in [41] show that, the phase transition from the normal (Non $S C$ ) to a superconducting state can be of the first or second order, depending on the value of the magnetic coupling constant $g$. The next two subsections are concerned with computation of the transition temperature $T_{\mathrm{c}}(\mathcal{H})$ for first and second order phase transitions.

\subsection{Second order phase transitions}

According to Section 3, (26) for the solution $\{\Delta \neq 0, \nu=$ $0\}$ reduces to the BCS gap equation

$$
\begin{aligned}
\Delta_{\mathrm{BCS}} & =\frac{1}{2} G_{0} \rho_{\mathrm{F}} \int_{-\delta}^{\delta} \frac{\Delta_{\mathrm{BCS}}}{E_{\mathrm{BCS}}} \tanh \left(\frac{1}{2} \beta E_{\mathrm{BCS}}\right) d \xi, \\
E_{\mathrm{BCS}} & =\sqrt{\xi^{2}+\Delta_{\mathrm{BCS}}^{2}} .
\end{aligned}
$$

The transition temperature $T_{\mathrm{c}}^{(\mathrm{BCS})}$ in BCS theory, is defined as the boundary of the region beyond which there is no real, positive $\Delta_{\mathrm{BCS}}$ satisfying $(56)$. Below $T_{\mathrm{c}}^{(\mathrm{BCS})}$ the solution $\Delta_{\mathrm{BCS}} \neq 0$ minimizes the free energy and the system is in superconducting phase. Therefore, $T_{\mathrm{c}}^{(\mathrm{BCS})}$ can be obtained from (56) with $\Delta_{\mathrm{BCS}}=0$, which yields [29]:

$$
T_{\mathrm{c}}^{(\mathrm{BCS})}=1.14 \delta \exp \left[-\left(G_{0} \rho_{\mathrm{F}}\right)^{-1}\right] .
$$

It should be possible to estimate the change in $T_{\mathrm{c}}^{(\mathrm{BCS})}$, since the density of states enters exponentially in (57). However, significant deviations from (57) were observed experimentally for a number of superconductors containing magnetic impurities. This inadequacy of (57) is most distinct for large values of impurity concentration. BCS 


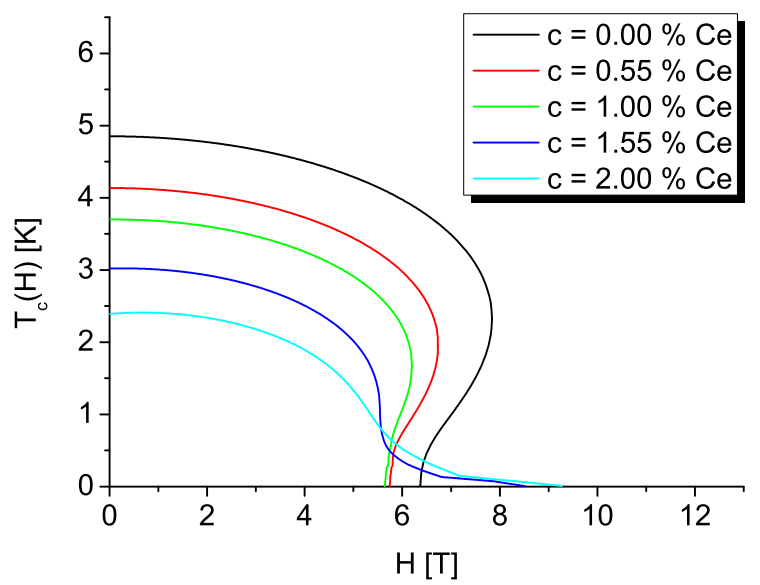

(a)

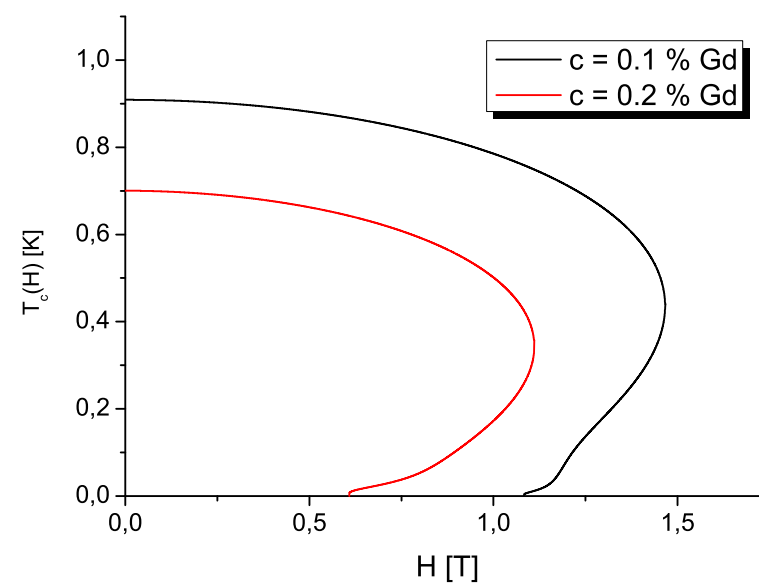

(b)

Fig. 8. $T_{\mathrm{c}}(\mathcal{H})$ graphs under varying impurity concentration for LaCe (a) and ThGd (b). The parameter values $\delta, g, g_{0}, g^{\prime}$, $G_{0} \rho_{F}$ and $M$ are collected in Table 3 .

theory is therefore incapable to describe the superconducting alloys.

Expression for transition temperature $T_{\mathrm{c}}$ of a superconducting alloy in the presence of an external magnetic field for 2nd order phase transition can be computed analogously as in BCS theory. To this end, it suffices to put $\Delta=0$ in equations (51) and (52). Thus, one obtains the following set of equations for $T_{\mathrm{c}}=1 /\left(k \beta_{\mathrm{c}}\right)$ :

$$
\begin{aligned}
2= & G_{0} \rho_{F} \int_{-\delta}^{\delta} \frac{d \xi}{|\xi|} \\
& \times \frac{\sinh \left(\beta_{\mathrm{c}}|\xi|\right)}{\cosh \left(\beta_{\mathrm{c}}|\xi|\right)+\cosh \left[\beta_{\mathrm{c}}\left(g M f_{2}^{(S)}\left(\zeta_{\mathrm{c}}\right)+\mu_{\mathrm{B}} \mathcal{H}\right)\right]} \\
\nu_{\mathrm{c}}= & \frac{c g}{M} \frac{\sinh \left[\beta_{\mathrm{c}}\left(g M f_{2}^{(S)}\left(\zeta_{\mathrm{c}}\right)+\mu_{\mathrm{B}} \mathcal{H}\right)\right]}{\cosh \left[\beta_{\mathrm{c}}\left(g M f_{2}^{(S)}\left(\zeta_{\mathrm{c}}\right)+\mu_{\mathrm{B}} \mathcal{H}\right)\right]+\cosh \left(\beta_{\mathrm{c}}|\xi|\right)} \\
& +f_{2}^{(S)}\left(\zeta_{\mathrm{c}}\right),
\end{aligned}
$$

where $\zeta_{\mathrm{c}}=\nu_{\mathrm{c}}-\frac{1}{2} g^{-1} g^{\prime} \mu_{\mathrm{B}} \tilde{\mathcal{H}}, \nu_{\mathrm{c}}=\nu\left(\beta_{\mathrm{c}}\right), S=1 / 2,7 / 2$.

Numerical analysis shows that in the low-temperature scale $\nu_{\mathrm{c}}(T)$ is almost independent in $T$, viz. $\nu_{\mathrm{c}}(T) \approx$ $\nu(0)=c g / M$. Accordingly, the set of equations (58) and (59) is solved under the assumption, that $\nu_{\mathrm{c}}=\mathrm{cg} / \mathrm{M}$.

The resulting solution for $T_{\mathrm{c}}(\mathcal{H})$ for $S=1 / 2,7 / 2$ under varying impurity concentration is depicted in Figure 8. The solution for $T_{\mathrm{c}}(\mathcal{H})$ for small $c$ is similar to the numerical result obtained by Sarma [57] of the system described by the Hamiltonian $H_{\mathrm{S}}=H_{\mathrm{BCS}}+\mu_{\mathrm{B}} \mathcal{H} \sigma_{z}$. His result for $T_{\mathrm{c}}^{(B C S)}(\mathcal{H})$ agrees qualitatively with $T_{\mathrm{c}}(\mathcal{H})$ graphs depicted in Figure 8, since the expression for $T_{\mathrm{c}}^{(B C S)}(\mathcal{H})$ obtained in [57] is of the similar form to (58) with $\zeta_{\mathrm{c}}=0$.

The $T_{\mathrm{c}}(\mathcal{H})$ graphs depicted in Figure $8 \mathrm{a}$ show two phase transitions for sufficiently small impurity concentration, i.e. $c \in(0$ at. $\%, 0.10$ at. $\%)$ and for $\mathcal{H} \in$
$(5.65 \mathrm{~T}, 7.8 \mathrm{~T})$. The first phase transition $(P \rightarrow S C)$ occurs at $T_{\mathrm{c} 1}(\mathcal{H})$ and the second phase transition (back to normal state) appears at $T_{\mathrm{c} 2}(\mathcal{H})$. In the case of LaCe, only one phase transition is present $(S C \rightarrow P)$ for higher concentrations, but superconductor accepts larger values of an external magnetic field. Furthermore, $T_{\mathrm{c}}(\mathcal{H})$ initially increases with $\mathcal{H}$.

The form of denominator on the right hand side of (58) suggests that, the perturbative effect of magnetic impurities can be compensated by an external magnetic field. It follows from the fact that $f_{2}^{(S)}\left(\zeta_{\mathrm{c}}\right)$ is odd function in $\zeta_{\mathrm{c}}$ and from a definition of $\zeta_{\mathrm{c}}$ parameter, which approaches negative values for sufficiently large $\mathcal{H}$. Thus, the magnetic field intensity, required for the full compensation of the perturbative effect of magnetic impurities on a BCS superconductor has the form:

$$
\mathcal{H}_{\mathrm{k}}=-\frac{g M}{\mu_{\mathrm{B}}} f_{2}^{(S)}\left(\zeta_{\mathrm{c}}\right) .
$$

This supposition has been verified for $g=0.95 \sqrt{\mathrm{eV}}$ and various impurity concentrations. The values of remaining parameters correspond to $\left(\mathrm{La}_{1-x} \mathrm{Ce}_{x}\right) \mathrm{Al}_{2}$ alloy (Tab. 3). The results, which are depicted in Figure 9, confirm the hypothesis of the Jaccarino-Peter compensation effect in the presented theoretical model. According to Figure 9, the function $T_{\mathrm{c} 1}(\mathcal{H})$ increases with $\mathcal{H}$ and after reaching a maximum at $\mathcal{H}=\mathcal{H}_{\mathrm{k}}$, decreases and finally falls to zero.

The number of magnetic moments, antiferromagnetically coupled to conduction fermions increases with increasing impurity concentration. It follows that an exchange field $\mathcal{H}_{\mathrm{J}}$ approaches larger values and as a result the compensation effect is present for larger values of $\mathcal{H}$. In the other words, the values of $\mathcal{H}$, where superconductivity appears, increase with $c$.

The $T_{\mathrm{c}}(\mathcal{H})$ graph depicted in Figure 9a resembles the dependence of superconducting transition temperature $T_{\mathrm{c}}$ on dopant concentration $p$, exhibited by high-temperature cuprates, e.g. $\mathrm{La}_{2-x} \mathrm{Sr}_{x} \mathrm{CuO}_{4}$ [58,59], $\mathrm{YBa}_{2} \mathrm{Cu}_{3} \mathrm{O}_{y}$ [60], 
Table 3. The parameter values.

\begin{tabular}{|c|c|c|c|c|c|c|c|c|}
\hline Alloy & $x$ & $\mathrm{c}[\%]$ & $M$ & $\delta[\mathrm{eV}]$ & $G_{0} \rho_{F}$ & $g^{\prime}$ & $g_{0}$ & $g[\sqrt{\mathrm{eV}}]$ \\
\hline \multirow{5}{*}{$\mathrm{LaCe}$} & & 0.00 & 0 & \multirow{5}{*}{0.01} & 0.3030 & \multirow{5}{*}{$10 / 7$} & \multirow{5}{*}{0.006} & 0.00 \\
\hline & & 0.55 & 2 & & 0.2890 & & & 0.080 \\
\hline & - & 1.00 & 4 & & 0.2800 & & & 0.095 \\
\hline & & 1.55 & 5 & & 0.2650 & & & 0.100 \\
\hline & & 2.00 & 6 & & 0.2500 & & & 0.105 \\
\hline \multirow{4}{*}{$\left(\mathrm{La}_{1-x} \mathrm{Ce}_{x}\right) \mathrm{Al}_{2}$} & 0.0010 & \multirow{4}{*}{-} & 1 & \multirow{4}{*}{0.01} & 0.2610 & \multirow{4}{*}{$10 / 7$} & \multirow{4}{*}{0.006} & 0.10 \\
\hline & 0.0019 & & 4 & & 0.2515 & & & 0.189 \\
\hline & 0.0028 & & 7 & & 0.2435 & & & 0.19 \\
\hline & 0.0057 & & 8 & & 0.2250 & & & 0.23 \\
\hline \multirow{3}{*}{ ThGd } & \multirow{3}{*}{ - } & 0.0 & 0 & \multirow{3}{*}{0.01} & 0.2200 & \multirow{3}{*}{2} & \multirow{3}{*}{0.004} & 0.00 \\
\hline & & 0.1 & 3 & & 0.2010 & & & 0.11 \\
\hline & & 0.2 & 5 & & 0.1915 & & & 0.18 \\
\hline
\end{tabular}

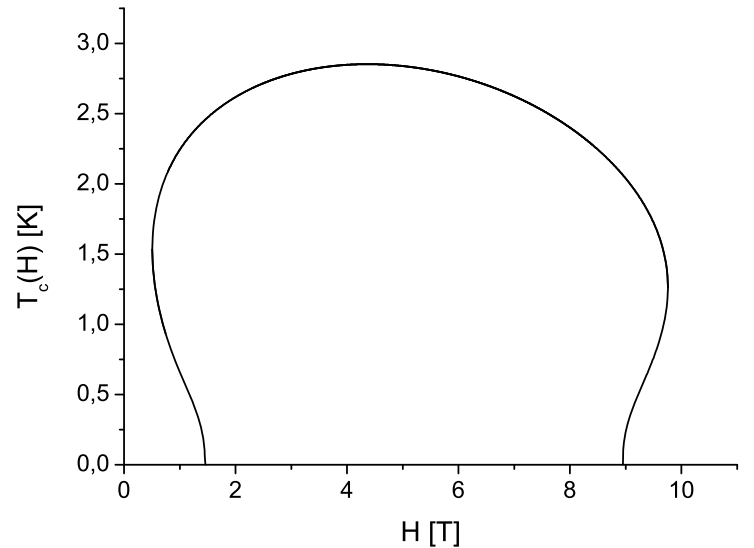

(a)

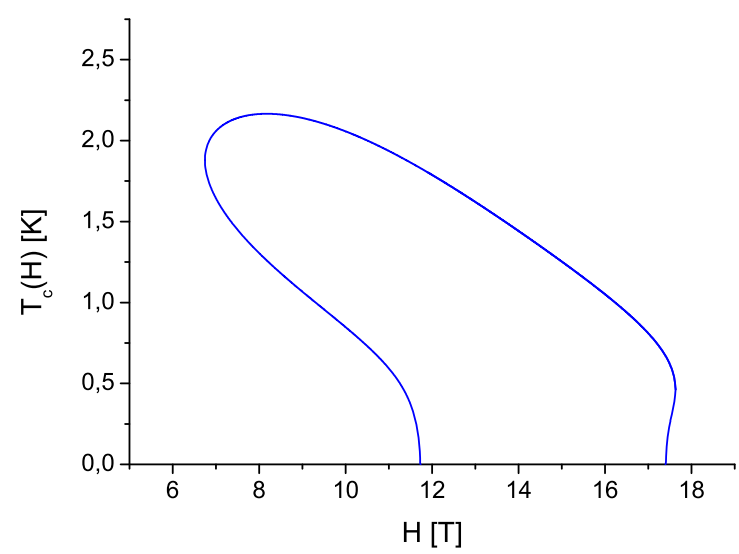

(c)

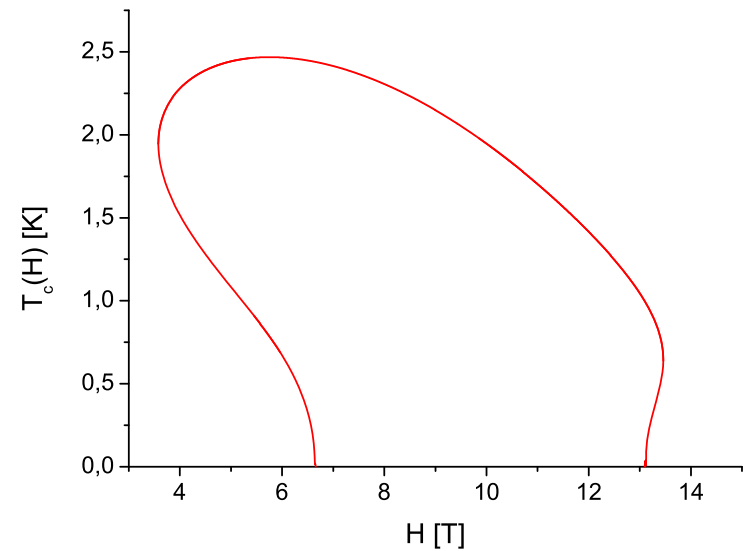

(b)

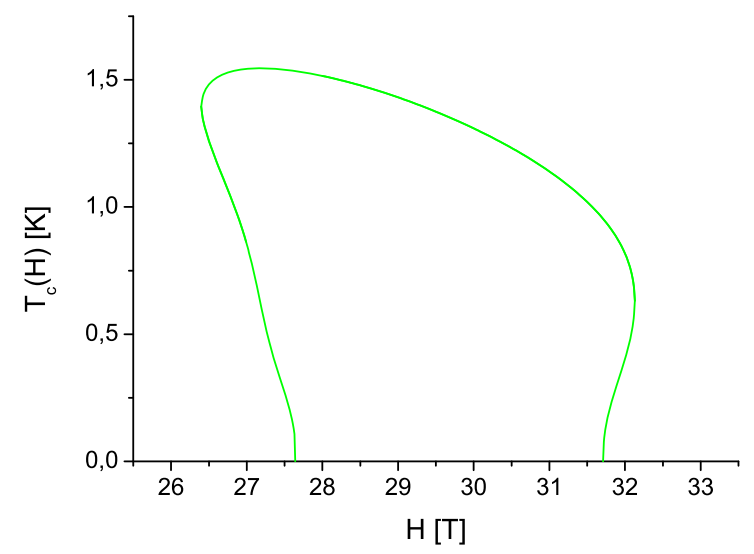

(d)

Fig. 9. $T_{\mathrm{c}}(\mathcal{H})$ graphs of $\left(\mathrm{La}_{1-x} \mathrm{Ce}_{x}\right) \mathrm{Al}_{2}$ for $g=0.95 \sqrt{\mathrm{eV}}$ and under varying impurity concentration: (a) $x=0.0010$, (b) $x=0.0019$, (c) $x=0.0028$, (d) $x=0.0057$. The values of the parameters $\delta, g_{0}, g^{\prime}, G_{0} \rho_{F}$ and $M$ are collected in Table 3 .

$\mathrm{Bi}_{2-x} \mathrm{~Pb}_{x} \mathrm{Sr}_{2} \mathrm{Ca}_{2} \mathrm{Cu}_{3} \mathrm{O}_{10}[60], \mathrm{Bi}_{2} \mathrm{Sr}_{2-x} \mathrm{La}_{x} \mathrm{CuO}_{6}[61]$ and iron-pnictides [5]. $T_{\mathrm{c}}(p)$ initially increases almost linearly in $p$ and after reaching a maximum at optimal doping level $p_{\text {opt }}$, decreases and finally falls to zero.

Analogous behavior of the superconducting transition temperature is observed, e.g. in CeRhIn ${ }_{5}$ [62], $\mathrm{CeCoIn}_{5}$ [63], when the superconductor is under pressure.

\subsection{First order phase transitions}

In the case, of first order phase transitions, the assumption that the gap parameter $\Delta$ vanishes at the transition temperature does not hold. According to the results obtained in [41], the superconducting transition temperature $T_{\mathrm{c}}$ possesses three solutions $\left(T_{\mathrm{c} 1} \geq T_{\mathrm{c} 2} \geq T_{\mathrm{c} 3}\right)$ for certain values of $g$ and $c$. These solutions can be determined 


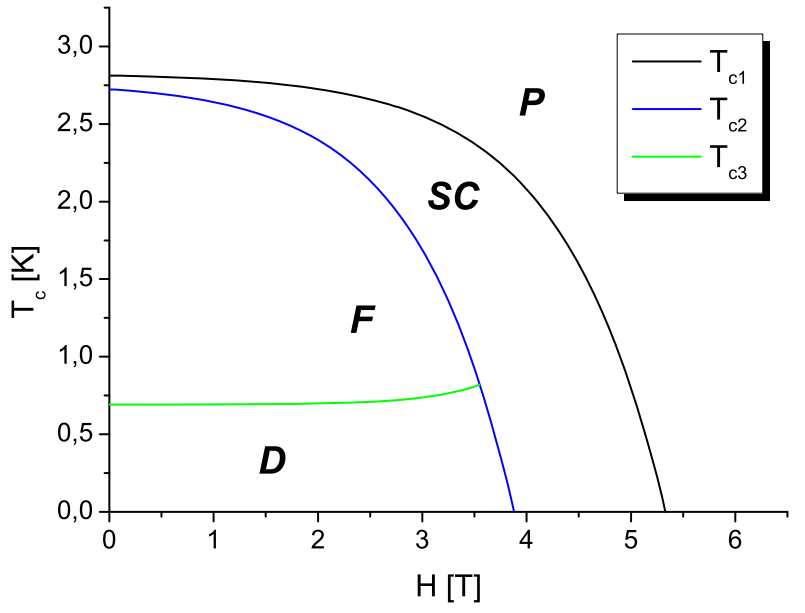

Fig. 10. $T_{\mathrm{c}}(\mathcal{H})$ graphs of $\left(\mathrm{La}_{1-x} \mathrm{Ce}_{x}\right) \mathrm{Al}_{2}$ for $g=0.5 \sqrt{\mathrm{eV}}$ and $x=0.0010$. The values of the parameters $\delta, g_{0}, g^{\prime}, G_{0} \rho_{F}$ and $M$ are collected in Table 3. For $\mathcal{H} \in(0 \mathrm{~T}, 3.55 \mathrm{~T})$ the system undergoes three phase transitions with decreasing temperature, $P \rightarrow S C \rightarrow F \rightarrow D$, showing the Jaccarino-Peter compensation effect.

numerically from the following equations:

$$
\begin{array}{ll}
T_{\mathrm{c} 1}: & F_{P}(\mathcal{H})-F_{S C}(\mathcal{H})=0, \\
T_{\mathrm{c} 2}: & F_{S C}(\mathcal{H})-F_{\Phi}(\mathcal{H})=0, \quad \Phi=D, F, \\
T_{\mathrm{c} 3}: & F_{F}(\mathcal{H})-F_{D}(\mathcal{H})=0 .
\end{array}
$$

The existence of $T_{\mathrm{c} 3}$ depends on the type of phase transition occuring at $T_{\mathrm{c} 2}$. If the system undergoes a phase transition to ferromagnetic phase at $T_{\mathrm{c} 2}$, then $T_{\mathrm{c} 3}>0$ for certain values of $g$. If $T_{\mathrm{c} 2}=T_{S C D}$, then $T_{\mathrm{c} 3}=0$ and the system does not reenter the superconducting phase $(S C$ or $D$ ).

The solution of equations (61)-(63) for the parameter values corresponding to $\left(\mathrm{La}_{-x} \mathrm{Ce}_{x}\right) \mathrm{Al}_{2}$ [41] are depicted in Figure 10. For $\mathcal{H} \in(0 \mathrm{~T}, 3.55 \mathrm{~T})$, three phase transitions $P \rightarrow S C \rightarrow F \rightarrow D$ with decreasing temperature are present. These phase transitions can be interpreted as Non $S C \rightarrow S C \rightarrow$ Non $S C \rightarrow S C$ transitions, i.e. the Jaccarino-Peter compensation effect, which has been experimentally observed in a number of superconducting magnetic alloys, e.g.: $\mathrm{Sn}_{x} \mathrm{Eu}_{1.2-x} \mathrm{Mo}_{6} \mathrm{~S}_{8}$ [64], $\mathrm{Eu}_{0.75} \mathrm{Sn}_{0.25} \mathrm{Mo}_{6} \mathrm{~S}_{7.2} \mathrm{Se}_{0.8}$ [25], CeCoIn [11], URhGe and UCoGe [15].

\section{Concluding remarks}

Our recent work on the phase diagrams of a BCS superconductor perturbed by a reduced $s$ - $d$ interaction [41] was continued to examine the critical magnetic field and the effect of an external magnetic field $\mathcal{H}$ on the superconducting transition temperature $T_{\mathrm{c}}$ of such system. The superconductivity enhancement, revealed by an increase of the critical magnetic field with decreasing temperature and increasing impurity concentration, has been found.
Good quantitative agreement of the resulting theoretical expressions for the critical magnetic field with experimental data was demonstrated for LaCe, ThGd and $\mathrm{SmRh}_{4} \mathrm{~B}_{4}$.

The numerical analysis of $T_{\mathrm{c}}(\mathcal{H})$ showed that the perturbative effect of magnetic impurities can be compensated by an external magnetic field, providing the evidence of the Jaccarino-Peter effect, which has been experimentally observed in a number of superconducting magnetic alloys, e.g.: $\mathrm{Sn}_{x} \mathrm{Eu}_{1.2-x} \mathrm{Mo}_{6} \mathrm{~S}_{8}$ [64], $\mathrm{Eu}_{0.75} \mathrm{Sn}_{0.25} \mathrm{Mo}_{6} \mathrm{~S}_{7.2} \mathrm{Se}_{0.8}$ [25]. The enhancement of superconductivity, displayed by an increase of the upper critical magnetic field with decreasing temperature due to the Jaccarino-Peter effect was discovered in $\mathrm{SmRh}_{4} \mathrm{~B}_{4}$ [36], $\mathrm{CeCoIn}_{5}$ [11], URhGe and UCoGe [15].

The enhancement of the critical magnetic field $\mathcal{H}_{\mathrm{c}}$ in magnetic superconductors, which is due to the interplay of the superconductivity and magnetic order, opens the door to their possible industrial applications. Since the upper critical magnetic field at absolute temperature of $\mathrm{PbMo}_{6} \mathrm{~S}_{8}$ magnetic superconductor, i.e. $\mathcal{H}_{\mathrm{c}}(0) \approx$ $60 \mathrm{~T}$ [9] doubly exceeds the corresponding value of the high-performance, low-temperature superconducting material $\mathrm{Nb}_{3} \mathrm{Sn}$, which is currently widely-exploited in power applications, e.g. in accelerators available at Fermilab, Brookhaven, DESY and CERN [2].

Author is grateful the Institute for Theoretical and Applied Physics for supporting his stay during the Eurasia-Pacific Summer School \& Workshop on Strongly Correlated Electrons (Turunc, Turkey, 6-17 August 2012), where part of this research was performed and presented. I also thank Prof. K. Machida and Dr. J. Mosqueira for their comments to the arXiv's version of this article.

\section{References}

1. H.K. Onnes, Commun. Phys. Lab. Univ. Leiden 120b, 122b, 124c (1911)

2. D. Larbalestier, A. Gurevich, D.M. Feldmann, A. Polyanskii, Nature 414, 368 (2001)

3. W.R. Hendee, C.J. Morgan, West J. Med. 141, 491 (1984)

4. J.G. Bednorz, K.A. Müller, Z. Phys. B 64, 189 (1986)

5. Y. Kamihara, T. Watanabe, M. Hirano, H. Hosono, J. Am. Chem. Soc. 130, 3296 (2008)

6. B.T. Matthias, H. Suhl, E. Corenzwit, Phys. Rev. Lett. 1, 93 (1958)

7. B.T. Matthias, E. Corenzwit, Phys. Rev. 100, 626 (1955)

8. B.T. Matthias, V.B. Compton, H. Suhl, E. Corenzwit, Phys. Rev. 115, 1597 (1959)

9. Superconductivity in Ternary Compounds I, Topics in Current Physics, edited by $\varnothing$. Fischer, M.B. Maple (Springer, Berlin, 1982), Vol. 32

10. Superconductivity in Ternary Compounds II, Topics in Current Physics, edited by M.B. Maple, Ø. Fischer (Springer, Berlin, 1982), Vol. 34

11. H.A. Radovan, N.A. Fortune, T.P. Murphy, E.C. Palm, S.T. Hannahs, S.W. Tozer, D. Hall, Nature 425, 51 (2003) 
12. M.B. Maple, E.D. Bauer, V.S. Zapf, J. Wosnitza, in Superconductivity, edited by K.H. Bennemann, J.B. Ketterson (Springer-Verlag, Berlin, Heidelberg, 2008), Vol. I, Chap. 13, and references therein

13. X. Chen, V.V. Struzhkin, Y. Yu, A.F. Goncharov, C. Lin, H. Mao, R.J. Hemle, Nature 466, 950 (2010)

14. H.J. Gardner, A. Kumar, L. Yu, P. Xiong, M.P. Warusawithana, L. Wang, O. Vafek, D.G. Schlom, Nat. Phys. 7, 895 (2011)

15. D. Aoki, T.D. Matsuda, F. Hardy, C. Meingast, V. Taufour, E. Hassinger, I. Sheikin, C. Paulsen, G. Knebel, H. Kotegawa, J. Flouquet, J. Phys. Soc. Jpn 80, SA008 (2011)

16. R.W. McCallum, D.C. Johnston, R.N. Shelton, M.B. Maple, Solid State Commun. 24, 391 (1977)

17. H.C. Hamaker, L.D. Woolf, H.B. MacKay, Z. Fisk, M.B. Maple, Solid State Commun. 31, 139 (1979)

18. M. Ischikawa, Ø. Fischer, Solid State Commun. 24, 747 (1977)

19. W.A. Fertig, D.C. Johnston, L.E. DeLong, R.W McCallum, M.B. Maple, B.T. Matthias, Phys. Rev. Lett. 38, 987 (1977)

20. M. Ischikawa, Ø. Fischer, Solid State Commun. 23, 37 (1977)

21. P. Fulde, R.A. Ferrell, Phys. Rev. 135, A550 (1964)

22. A.I. Larkin, Y.N. Ovchinnikov, Sov. Phys. J. Exp. Thoer. Phys. 20, 762 (1965)

23. A. Bianchi, R. Movshovich, N. Oeschler, P. Gegenwart, F. Steglich, J.D. Thompson, P.G. Pagliuso, J.L. Sarrao, Phys. Rev. Lett. 89, 137002 (2002)

24. V.J. Jaccarino, M. Peter, Phys. Rev. Lett. 9, 290 (1962)

25. H.W. Meul, C. Rossel, M. Decroux, O. Fischer, G. Remenyi, A. Briggs, Phys. Rev. Lett. 53, 497 (1984)

26. K. Nakamura, Prog. Theor. Phys. 22, 156 (1959)

27. H. Suhl, B.T. Matthias, Phys. Rev. 114, 977 (1959)

28. T. Kasuya, Prog. Theor. Phys. 16, 45 (1956)

29. J. Bardeen, L.N. Cooper, J.R. Schrieffer, Phys. Rev. 108, 1175 (1957)

30. C.A. Balseiro, L.M. Falicov, Phys. Rev. B. 19, 2548 (1979)

31. A.A. Abrikosov, L.P. Gor'kov, Zh. Eksp. Teor. Fiz. 39, $1781(1960)$

32. F. Reif, M.A. Woolf, Phys. Rev. Lett. 9, 315 (1962)

33. P.M. Chaikin, T.W. Mihalisin, Phys. Rev. B 6, 839 (1972)

34. R.J. Delfs, B.J. Beaudry, D.K. Finnemore, Phys. Rev. B 11, $4212(1975)$

35. T.V. Ramakrishnan, C.V. Varma, Phys. Rev. B 24, 137 (1981)

36. H.C. Hamaker, D. Woolf, H.B. MacKay, Z. Fisk, M.B. Maple, Solid State Commun. 31, 139 (1979)

37. K. Machida, K. Nokura, T. Matsubara, Phys. Rev. B 22, 2307 (1980)

38. F. Soto, L. Cabo, J. Mosqueira, M.V. Ramallo, J.A. Veira, F. Vidal, Europhys. Lett. 73, 587 (2006)
39. A.G. Kozorezov, A.A. Golubov, J.K. Wigmore, D. Martin, P. Verhoeve, R.A. Hijmering, I. Jerjen, Phys. Rev. B 78, 174501 (2008)

40. A.V. Balatsky, I. Vekhter, J.X. Zhu, Rev. Mod. Phys. 78, $373(2006)$

41. D. Borycki, J. Maćkowiak, Supercond. Sci. Technol. 24, 035007 (2011)

42. M. Sigrist, K. Ueda, Rev. Mod. Phys. 63, 239 (1991)

43. R. Feynman, in Statistical Mechanics, a Set of Lectures, Frontiers in Physics (W.A. Benjamin, London, Amsterdam, 1972), Sect. 2.11

44. N.N. Bogolyubov, Nuovo Cim. 7, 794 (1958)

45. J.G. Valatin, Nuovo Cim. 7, 843 (1958)

46. G. Rickayzen, Theory of superconductivity (Interscience Publishers, NY, 1965)

47. T. Sugawara, H. Eguchi, J. Phys. Soc. Jpn 23, 965 (1967)

48. W.R. Decker, D.T. Peterson, D.K. Finnemore, Phys. Rev. Lett. 18, 899 (1967)

49. J. Maćkowiak, Open Sys. Inf. Dyn. 14, 229 (2007)

50. J. Maćkowiak, D. Borycki, Mod. Phys. Lett. B 24, 2131 (2010)

51. Ø. Fischer, M. Decroux, S. Roth, R. Chevrel, M. Sergent, J. Phys. C 8, L474 (1975)

52. B.S. Chandrasekhar, Appl. Phys. Lett. 1, 7 (1962)

53. A.M. Clogston, Phys. Rev. Lett. 9, 266 (1962)

54. The Physics of Superconductors I, II, edited by K.H. Bennemann, J.B. Ketterson (Springer, Berlin, New York, 2004)

55. D.C. Mattis, in The Theory of Magnetism II (Springer, Berlin, New York, 1985), p. 15

56. W. Felsch, K. Winzer, G.V. Minnigerode, Z. Phys. B 21, 151 (1975)

57. G. Sarma, J. Phys. Chem. Solids 24, 1029 (1963)

58. J.T. Markert, Y. Dalichaouch, M.B. Maple, in Physical Properties of High Temperature Superconductors I, edited by D.M. Ginsberg (World Scientific, Singapore, 1989)

59. K. Yamada et al., Phys. Rev. B 57, 6165 (1998)

60. Y.J. Uemura et al., Phys. Rev. Lett. 62, 2317 (1989)

61. Y. Ando, T. Murayama, Phys. Rev. B 60, R6991 (1999)

62. Y. Kitaoka et al., J. Phys. Chem. Solids 63, 1141 (2002)

63. V. Sidorov, M. Nicklas, P.G. Pagliuso, J.L. Sarrao, Y. Bang, A.V. Balatsky, J.D. Thompson, Phys. Rev. Lett. 89, 157004 (2002)

64. S.A. Wolf, W.W. Fuller, C.Y. Huang, D.W. Harrison, H.L. Luo, S. Maekawa, Phys. Rev. B 25, 1990 (1982)

Open Access This is an open access article distributed under the terms of the Creative Commons Attribution License (http://creativecommons.org/licenses/by/4.0), which permits unrestricted use, distribution, and reproduction in any medium, provided the original work is properly cited. 